\title{
1 The temporal and spectral characteristics of expectations and 2 prediction errors in pain and thermoception
}

3 Andreas Strube ${ }^{1}$, Michael Rose ${ }^{1}$, Sepideh Fazeli ${ }^{1} \&$ Christian Büchel $^{1}$

4 1Department of Systems Neuroscience, University Medical Center Hamburg-Eppendorf, 20246 Hamburg, Germany

6 Corresponding Author: Christian Büchel

7

8

9

10

11

12

13 Keywords:
Department of Systems Neuroscience University Medical Center Hamburg-Eppendorf 20246 Hamburg, Germany Phone: +4940741057 email: buechel@uke.de 14 


\section{Abstract}

16 In the context of a generative model, such as predictive coding, pain and heat perception 17 can be construed as the integration of expectation and input with their difference 18 denoted as a prediction error. In a previous neuroimaging study (Geuter et al., 2017) we 19 observed an important role of the insula in such a model, but could not establish its 20 temporal aspects. Here we employed electroencephalography to investigate neural 21 representations of predictions and prediction errors in heat and pain processing. Our 22 data show that alpha-to-beta activity was associated with stimulus intensity expectation, 23 followed by a negative modulation of gamma band activity by absolute prediction errors. 24 This is in contrast to prediction errors in visual and auditory perception, which are 25 associated with increased gamma band activity, but is in agreement with observations in 26 working memory and word matching, which show gamma band activity for correct, 27 rather than violated predictions.

\section{Introduction}

29 It has been shown that physically identical nociceptive input, can lead to variable 30 sensations of pain, depending on contextual factors (Tracey and Mantyh, 2007). In particular, attention, reappraisal and expectation are core mechanisms that influence how nociception leads to pain (Wiech et al., 2008). A clinically important example of how expectations can shape pain processing is placebo hypoalgesia: pain relief mediated by expectation and experience - in the absence of active treatment (Petrovic et al., 2002; Wager et al., 2004; Colloca and Benedetti, 2005; Bingel et al., 2006; Atlas and Wager, 2012; Anchisi and Zanon, 2015).

In the context of a generative model of pain, it has been proposed that pain perception can be seen as the consequence of an integration of expectations with nociception (Büchel et al., 2014; Wiech, 2016; Ongaro and Kaptchuk, 2019). In this framework, expectations are integrated with incoming nociceptive information and both are weighted by their relative precision (Grahl et al., 2018) to form a pain percept. This can be seen in analogy to ideas in multisensory integration (Ernst and Banks, 2002). Expectations or predictions and resulting prediction errors also play a key role in 44 generative models such as predictive coding (Huang and Rao, 2011). In essence, this 45 framework assumes that neuronal assemblies implement perception and learning by 
46 constantly matching incoming sensory data with the top-down predictions of an internal 47 or generative model (Knill and Pouget, 2004; Huang and Rao, 2011; Clark, 2013).

48 Basically, minimizing prediction errors allows systems to resist their tendency to 49 disorder by the creation of models with better predictions regarding the sensory 50 environment, leading to a more efficient encoding of information (Friston, 2010).

51 EEG correlates of nociceptive skin stimulation have been widely investigated. Generally, 52 phasic gamma activity has been associated with stimulus intensity over the sensory 53 cortex where the amplitudes of pain-induced gamma oscillations increase with objective 54 stimulus intensity and subjective pain intensity (Gross et al., 2007; Hauck et al., 2007; 55 Zhang et al., 2012; Rossiter et al., 2013; Tiemann et al., 2015). Additionally, pain-related 56 gamma band oscillations have been linked to the insular cortex as well as temporal and frontal regions using depth electrodes in epilepsy patients (Liberati et al., 2018). In tonic painful heat stimulation, medial prefrontal gamma activity has been observed (Schulz et al., 2015). In addition, gamma activity is enhanced by attention in human EEG experiments in visual (Gruber et al., 1999), auditory (Tiitinen et al., 1993; Debener et al., 2003) and sensorimotor processing (i.e. tactile stimuli) (Bauer et al., 2006) as well as in nociception (Hauck et al., 2007, 2015; Tiemann et al., 2010).

Pain-related alpha-to-beta band oscillations are typically found to be suppressed with higher stimulus intensity (Mouraux et al., 2003; Ploner et al., 2006; May et al., 2012; Hu et al., 2013), which is enhanced by attention (May et al., 2012) and (placebo) expectation (Huneke et al., 2013; Tiemann et al., 2015; Albu and Meagher, 2016). Interestingly, prestimulus theta (Taesler and Rose, 2016) as well as pre-stimulus alpha and gamma activity (Tu et al., 2016) can affect subsequent pain processing. Specifically, trials with smaller pre-stimulus alpha and gamma oscillations were perceived as more painful, suggesting a negative modulation of subsequent pain perception (Tu et al., 2016).

71 Cued pain paradigms (Atlas et al., 2010) have been used to generate expectations and 72 prediction errors. Previous fMRI studies have suggested an important role of the 73 anterior insular cortex for mediating expectation effects and the integration of prior 74 expectation and prediction errors in the context of pain (Ploghaus et al., 1999; Koyama 75 et al., 2005; Atlas et al., 2010; Geuter et al., 2017; Fazeli and Büchel, 2018). These studies 76 have revealed that neuronal signals in the anterior insula represent predictions and 77 prediction errors with respect to pain, which in theory would allow the combination of 
78 both terms as required for predictive coding (Büchel et al., 2014; Ongaro and Kaptchuk,

79 2019). However, in fMRI studies predictions and prediction errors cannot be temporally

80 dissociated due to the low temporal resolution of the method. To investigate this further,

81 we conducted a cue based pain experiment using EEG to achieve high temporal and

82 spectral resolution of predictions and prediction error processes in the context of pain.

83 In this experiment $(\mathrm{N}=29)$ we employed contact heat stimuli with three different 84 intensities (low heat, medium heat and high heat), preceded by a visual cue indicating 85 the upcoming intensity (Figure 1). To generate prediction errors, the modality (picture 86 or heat) was correctly cued only in $70 \%$ of all trials and stimulus intensities were 87 correctly cued only in $60 \%$ of all trials. We then investigated oscillatory activity related 88 to stimulus intensity, expectation and prediction errors (Figure 2).

89
Trial Structure

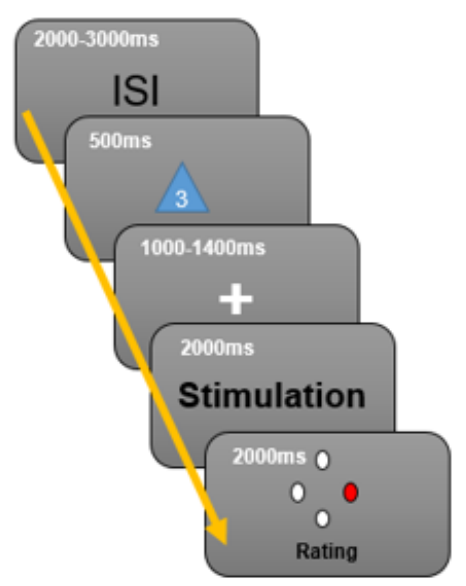

Cue-Stimulus Contingencies

\begin{tabular}{|c|c|c|c|c|c|c|}
\hline & $42{ }^{\circ} \mathrm{C}$ & $46{ }^{\circ} \mathrm{C}$ & $48{ }^{\circ} \mathrm{C}$ & $\begin{array}{l}\text { Picture } \\
\text { Level 1 }\end{array}$ & $\begin{array}{l}\text { Picture } \\
\text { Level 2 }\end{array}$ & $\begin{array}{l}\text { Picture } \\
\text { Level 3 }\end{array}$ \\
\hline 1 & $7.0 \%$ & $2.3 \%$ & $2.3 \%$ & $3.0 \%$ & $1.0 \%$ & $1.0 \%$ \\
\hline 2 & $2.3 \%$ & $7.0 \%$ & $2.3 \%$ & $1.0 \%$ & $3.0 \%$ & $1.0 \%$ \\
\hline 3 & $2.3 \%$ & $2.3 \%$ & $7.0 \%$ & $1.0 \%$ & $1.0 \%$ & $3.0 \%$ \\
\hline 1 & $3.0 \%$ & $1.0 \%$ & $1.0 \%$ & $7.0 \%$ & $2.3 \%$ & $2.3 \%$ \\
\hline 2 & $1.0 \%$ & $3.0 \%$ & $1.0 \%$ & $2.3 \%$ & $7.0 \%$ & $2.3 \%$ \\
\hline 3 & $1.0 \%$ & $1.0 \%$ & $3.0 \%$ & $2.3 \%$ & $2.3 \%$ & $7.0 \%$ \\
\hline
\end{tabular}

Figure 1. Left: Graphical representation of the trial structure. Each trial started with the presentation of a cue, indicating the stimulus intensity and modality of the following stimulus. After a jittered phase where only the fixation cross was shown, the stimulus (visual or thermal) was presented. A rating phase (1-4) of the stimulus aversiveness followed. Right: Contingency table for all conditions for each cue-stimulus combination. Note that percentages are for all trials, therefore each row adds up to $1 / 6$ ( 6 different cues). 


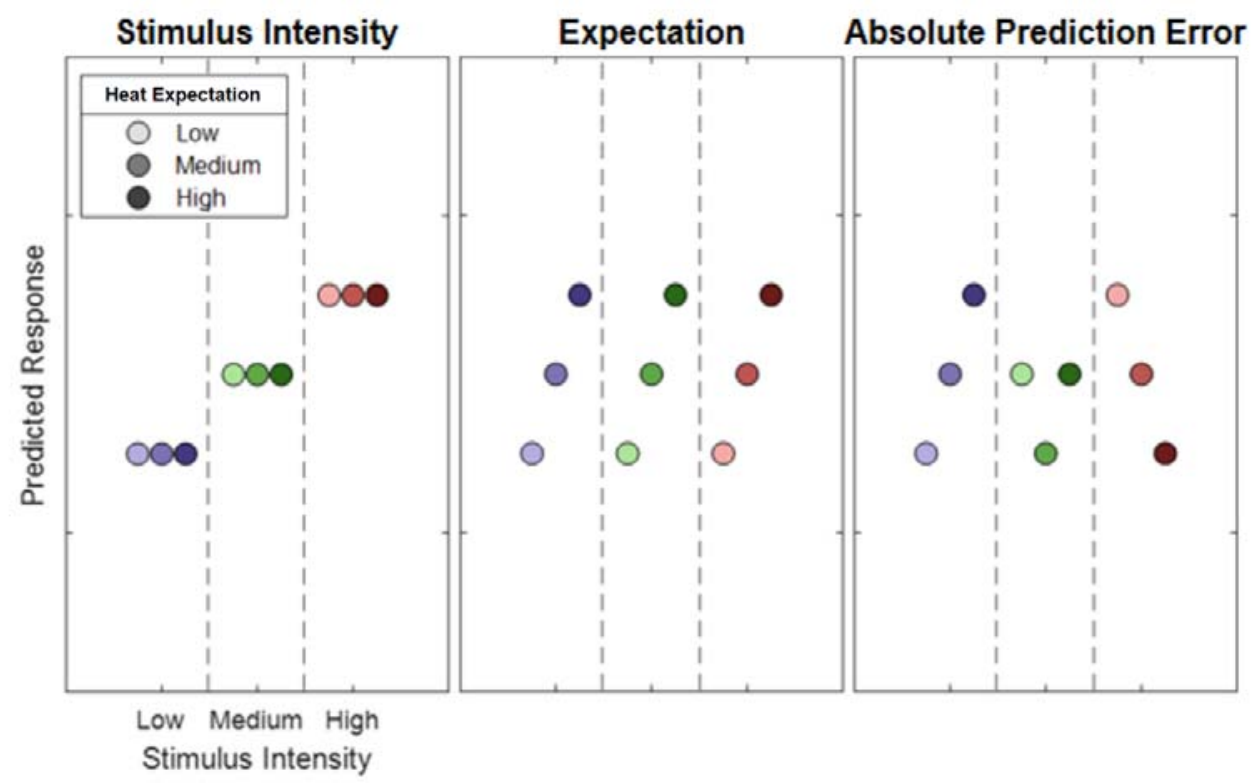

97 Figure 2. Hypothetical response patterns based on Stimulus Intensity (left), Expectation 98 (middle) and Absolute Prediction Error (right). The y-axis represents a hypothetical response variable (e.g. EEG power). Each dot represents a different condition for each stimulus-cue combination. Blue colors represent low heat conditions, green colors represent medium heat

101 conditions and red colors represent high heat conditions. Color intensities depict expectation 102 level.

103 Based on previous data, we (i) hypothesized that expectation signals should temporally 104 precede prediction error signals. (ii) Based on the functional neuroanatomy of cortical 105 microcircuits (Bastos et al., 2012), with feedforward connections predominately originating from superficial layers and feedback connections from deep layers, we expect that prediction error signals should be related to higher frequencies (e.g. gamma band) than prediction signals (Todorovic et al., 2011; Arnal and Giraud, 2012).

\section{Methods}

\section{Participants}

111 We investigated 35 healthy male participants (mean 26, range: 18-37 years), who were 112 paid as compensation for their participation. Applicants were excluded if one of the 113 following exclusion criteria applied: neurological, psychiatric, dermatological diseases, 114 pain conditions, current medication, or substance abuse. All volunteers gave their 115 informed consent. The study was approved by the Ethics board of the Hamburg Medical 116 Association. Of 35 participants, data from six participants had to be excluded from the 117 final EEG data analysis due to technical issues during the EEG recording (i.e.: the data of 
118 the excluded participants were contaminated with excessive muscle and/or technical 119 artifacts) leaving a final sample of 29 participants. The sample size was determined 120 according a power calculation ( $G *$ Power V 3.1.9.4) based on Geuter et al., 2017. For the 121 left anterior insula (fMRI; Table 1 in Geuter et al., 2017) we observed an effect size of 122 partial eta squared of 0.17 and an effect size of 0.22 for the right anterior insula (Cue $\mathrm{x}$ 123 stimulus interaction). Using a power of (1-beta) of 0.95 and an alpha level of 0.05 and 124 assuming a low correlation (0.1) between repeated measures, this leads to a sample size 125 of 25 assuming the weaker effect in the left insula.

\section{Stimuli and Task}

127 Stimulus properties were chosen to be identical to a previous fMRI study of predictive 128 coding in pain where both expectation and absolute prediction error effects were 129 observed (Fazeli and Büchel, 2018). Thermal stimulation was performed using a $30 \times 30$ $130 \mathrm{~mm}^{2}$ Peltier thermode (CHEPS Pathway, Medoc) at three different intensities: low heat $131\left(42^{\circ} \mathrm{C}\right)$, medium heat $\left(46^{\circ} \mathrm{C}\right)$, and high heat $\left(48^{\circ} \mathrm{C}\right)$ at the left radial forearm. These three 132 temperatures cover a large range of temperatures associated with nociception. The 133 lowest temperature was set at $42^{\circ} \mathrm{C}$ to ensure a temperature above the median 134 threshold of heat sensitive C-fiber nociceptors which have a median heat threshold of $13541^{\circ} \mathrm{C}$ (Treede et al., 1995). The baseline temperature was set at $33^{\circ} \mathrm{C}$ and the rise rate to $13640^{\circ} \mathrm{C} / \mathrm{s}$. After two blocks, the stimulated skin patch was changed to avoid sensitization.

137 Aversive pictures were chosen from the International Affective Picture System (IAPS) 138 (Lang et al., 2008) database at three different levels of aversiveness. The images 139 presented during the EEG experiment had three levels of valence of which the low 140 valence category had valence values of $2.02 \pm 0.05$ (mean \pm standard error), the medium 141 valence category had valence values of $4.06 \pm 0.02$ (mean \pm standard error) and the high 142 valence category had valence values of $5.23 \pm 0.01$ (mean \pm standard error).

143 Prior to each picture or heat stimulus, a visual cue was presented. The color of the cue 144 (triangle) indicated (probabilistically) the modality of the stimulus (orange for picture 145 and blue for heat). A white digit written inside of each triangle indicated 146 (probabilistically) the intensity of the subsequent stimulus (1, 2 and 3 for low, medium 147 and high intensity). During the whole trial, a centered fixation cross was presented on 148 the screen. 
149 Each trial began with the presentation of the cue for $500 \mathrm{~ms}$ as an indicator for the 150 modality and intensity of the subsequently presented stimulus. The modality was 151 correctly cued in $70 \%$ of all trials by the color of the triangle. In $60 \%$ of all trials, the 152 stimulus intensity was correctly indicated by the digit within the triangle (see Figure 1 153 for an overview of all cue contingencies).

154 Before the presentation of the stimulus, there was a blank period with a variable time 155 frame between $1000 \mathrm{~ms}$ and $1400 \mathrm{~ms}$. Then, the visual or thermal stimulus was 156 presented for a duration of two seconds. The visual stimulus was centered on the screen 157 and allowed the participant to perceive the stimulus without eye movements. Right after 158 the termination of the stimulus, subjects were asked to rate the aversiveness of the 159 stimulus on a four point rating scale, where 1 was labeled as "neutral" and 4 was labeled 160 as "very strong". Ratings were performed using a response box operated with the right 161 hand (see Figure 1 for a visualization of the trial structure).

162 In addition, four catch trials were included in each block. Subjects were asked to report 163 the preceding cue in terms of their information content of the modality and intensity 164 within 8 s and no stimulation was given in these trials.

165 Trials were presented in four blocks. Each block consisted of 126 trials and four catch 166 trials and lasted about 15 minutes. The trial order within each block was 167 pseudorandomized. The order of blocks was randomized across subjects. The whole EEG 168 experiment including preparation and instructions lasted for about three hours.

169 Prior to the actual EEG experiment, subjects participated in a behavioral training 170 session. During this session, participants were informed about the procedure and gave 171 their written informed consent. The behavioral training session was implemented to 172 avoid learning effects associated with the contingencies between the cues and the 173 stimuli during the EEG session. Between two and three blocks were presented during 174 the training session (without electrophysiological recordings). The experimenter 175 assessed the performance after each block based on the percentage of successful catch 176 trials and the ability to distinguish the three levels of aversiveness of each modality. The 177 training session was terminated after the second block if participants were able to 178 successfully label cues in $75 \%$ of the catch trials within the second block. 


\section{EEG Data Acquisition}

180 EEG data were acquired using a 64-channel Ag/AgCl active electrode system (ActiCap64;

181 BrainProducts) placed according to the extended 10-20 system (Klem et al., 1999). Sixty

182 electrodes were used of the most central scalp positions. The EEG was sampled at

$183500 \mathrm{~Hz}$, referenced at FCz and grounded at Iz. For artifact removal, a horizontal, bipolar

184 electrooculogram (EOG) was recorded using two of the remaining electrodes and

185 placing them on the skin approximately $1 \mathrm{~cm}$ left from the left eye and right from the

186 right eye at the height of the pupils. One vertical electrooculogram was recorded using

187 one of the remaining electrodes centrally approx. $1 \mathrm{~cm}$ beneath the left eye lid and

188 another electrode was fixated on the neck at the upper part of the left trapezius muscle

189 (Musculus trapezius) to record an electromyogram (EMG).

\section{EEG Preprocessing}

191 The data analysis was performed using the Fieldtrip toolbox for EEG/MEG-analysis

192 (Oostenveld et al., 2011, Donders Institute for Brain, Cognition and Behaviour, Radboud

193 University Nijmegen, the Netherlands. See http://www.ru.nl/neuroimaging/fieldtrip).

194 EEG data were epoched and time-locked to the stimulus onset using the electrical trigger

195 signaling the thermode to start the temperature rise of a given heat trial. Each epoch

196 was centered (subtraction of the temporal mean) and detrended and included a time

197 range of $3410 \mathrm{~ms}$ before and $2505 \mathrm{~ms}$ after trigger onset.

198 The data was band-pass filtered at 1-100Hz, Butterworth, 4th order. EEG epochs were

199 then visually inspected and trials contaminated by artifacts due to gross movements or

200 technical artifacts were removed. Subsequently, trials contaminated by eye-blinks and

201 movements were corrected using an independent component analysis (ICA) algorithm

202 (Makeig et al., 1996; Jung et al., 2000). In all datasets, individual eye movements,

203 showing a large EOG channel contribution and a frontal scalp distribution, were clearly

204 seen in the removed independent components. Additionally, time-frequency

205 decomposed ICA data were inspected at a single trial level, after z-transformation (only

206 for artifact detection purposes) based on the mean and the standard deviation across all

207 components separately for each frequency from 31 to $100 \mathrm{~Hz}$. Time-Frequency

208 representations were calculated using a sliding window multi-taper analysis with a

209 window of $200 \mathrm{~ms}$ length, which was shifted over the data with a step size of $20 \mathrm{~ms}$ with

210 a spectral smoothing of $15 \mathrm{~Hz}$. Artifact components or trials were easily visible and were 
211 compared with the raw ICA components. Specifically, single and separate muscle spikes 212 were identified as columns or "clouds" in time-frequency plots. Using this procedure, up

213 to 30 components were removed before remaining non-artifactual components were 214 back-projected and resulted in corrected data. Subsequently, the data was re-referenced

215 to a common average of all EEG channels and the previous reference channel FCz was 216 re-used as a data channel (see Figure 1, figure supplement 1 for a summary of rejected 217 components per participant).

218 Before time-frequency transformations for data analysis were performed on the cleaned 219 data set, the time axis of single trials were shifted to create cue-locked and stimulus220 locked data. Cue-locked data uses the onset of the cue as $t=0$. Stimulus-locked data 221 takes the ramp up period of the thermode into account and sets $t=0$ to the time point 222 when the thermode reached the target temperature (225ms, 325ms and 375 after 223 trigger onset for low, medium and high heat conditions, respectively). Frequencies up to $22430 \mathrm{~Hz}$ ( 1 to $30 \mathrm{~Hz}$ in $1 \mathrm{~Hz}$ steps) were analyzed using a sliding Hanning-window Fourier 225 transformation with a window length of $300 \mathrm{~ms}$ and a step-size of $50 \mathrm{~ms}$. For the analysis 226 of frequencies higher than $30 \mathrm{~Hz}$ (31 to $100 \mathrm{~Hz}$ in $1 \mathrm{~Hz}$ steps) spectral analyses of the EEG 227 data were performed using a sliding window multi-taper analysis. A window of $200 \mathrm{~ms}$ 228 length was shifted over the data with a step size of $50 \mathrm{~ms}$ with a spectral smoothing of 15 229 Hz. Spectral estimates were averaged for each subject over trials. Afterwards, a z230 baseline normalization was performed based on a $500 \mathrm{~ms}$ baseline before cue onset. For 231 cue-locked data, a time frame ranging from $-650 \mathrm{~ms}$ to $-150 \mathrm{~ms}$ was chosen as a baseline. 232 A distance from the cue onset to the baseline period of $150 \mathrm{~ms}$ was set because of the 233 half-taper window length of $150 \mathrm{~ms}$, i.e. data points between $-150 \mathrm{~ms}$ and $0 \mathrm{~ms}$ are 234 contaminated by the onset of the cue. For stimulus-locked trials, a variable cue duration 235 (1500-1900ms) and a variable stimulus offset based on the ramp-up time (225-375ms) 236 were additionally taken into account, resulting in an according baseline from $-2950 \mathrm{~ms}$ to $237-2450 \mathrm{~ms}$ from stimulus onset. For the baseline correction of time-frequency data, the 238 mean and standard deviation were estimated for the baseline period (for each subject239 channel-frequency combination, separately). The mean spectral estimate of the baseline 240 was then subtracted from each data point, and the resulting baseline-centered values 241 were divided by the baseline standard deviation (classical baseline normalization 242 additive model; see Grandchamp and Delorme, 2011). 


\section{Predictive Coding Model}

244 Similar to a previous fMRI study (Fazeli and Büchel, 2018), our full model included three

245 experimental within-subject factors (see Figure 2). The stimulus intensity factor (INT)

246 models the measured response with a simple linear function of the stimulus intensity (-

247 1, 0 and 1 for low, medium and high intensities, respectively). The expectation (EXP)

248 factor was defined (see Figure 2; center column) linearly from the intensity predicted by

249 the cue. Again, conditions with a low intensity cue were coded with a -1 , conditions with

250 a medium intensity cue with a 0 and conditions with a high intensity cue with a 1 . The

251 absolute prediction error factor (PE) resulted from the absolute difference of the

252 expectation and actual stimulus intensity (see Figure 2; right column).

253 We also investigated a signed PE. However, it should be noted that such a term is not 254 orthogonal to the expectation factor. However, assuming that an expectation can only be 255 observed after the cue and a PE after the nociceptive stimulus, we were able to test for a 256 signed PE during the heat phase. Also, we considered a one-sided prediction error 257 factor, where a prediction error is only signaled when the stimulus is more intense as 258 expected, which is motivated by previous work (Egner et al., 2010; Summerfield and de 259 Lange, 2014; Geuter et al., 2017).

260 As the lowest stimulus intensity was often perceived as non-painful, we additionally

261 performed an analysis only with medium and high stimulus intensities. Accordingly, the 262 lowest stimulus intensity $\left(42^{\circ} \mathrm{C}\right)$ were excluded in an additional repeated measures

263 ANOVA analysis for this purpose (which will be referred to as the reduced pain model).

\section{Behavioral Aversiveness Ratings}

265 Behavioral aversiveness ratings were averaged for all $3 \times 3$ cue-stimulus combinations 266 over each participant, resulting in a $29 \times 9$ matrix (subject $\mathrm{x}$ condition) for the full model 267 and a 29x6 matrix for the reduced pain model. We tested for main effects across 268 stimulus intensity, expectation, as well as prediction error using a repeated measures 269 ANOVA as implemented in MATLAB (see fitrm and ranova; version 2020a, The 270 MathWorks). 


\section{EEG data analysis}

272 All statistical tests in electrode space were corrected for multiple comparisons using 273 non-parametrical permutation tests of clusters (Maris \& Oostenveld, 2007). Cluster 274 permutation tests take into account that biological processes are not strictly locked to a 275 single frequency or time point and that activity could be picked up by multiple 276 electrodes. Cluster permutation tests are specifically useful for explorative testing, as 277 explained by Maris \& Oostenveld (2007). While prior hypotheses could have been 278 formulated regarding the spatial, temporal and spectral characteristics of brain 279 responses associated with the intensity of thermal stimulation and regions-of-interest 280 could have been described, variations in the present design could be related to temporal 281 and spectral differences compared to other studies which would be taken into account 282 using non-parametric cluster permutation testing.

283 We wanted to explore positive and negative time-frequency patterns associated with 284 our variations of stimulus intensity, expectation and absolute prediction errors using a 285 repeated measures ANOVA. A statistical value corresponding to a p-value of .05 $(\mathrm{F}(1,28)$ $286=4.196)$ obtained from the repeated measures ANOVA F-statistics of the respective 287 main effect was used for clustering. Samples (exceeding the threshold of $F(1,28)=$ 288 4.196) were clustered in connected sets on the basis of temporal (i.e. adjacent time 289 points), spatial (i.e. neighboring electrodes) and spectral (i.e. +/- 1Hz) adjacency. 290 Further, clustering was restricted in a way that only samples were included in a cluster 291 which had at least one significant neighbor in electrode space, i.e. at least one 292 neighboring channel also had to exceed the threshold for a sample to be included in the 293 cluster. Neighbors were defined by a template provided by the Fieldtrip toolbox 294 corresponding to the used EEG montage.

295 Subsequently, a cluster value was defined as the sum of all statistical values of included 296 samples. Monte Carlo sampling was used to generate 1000 random permutations of the 297 design matrix and statistical tests were repeated in time-frequency space with the 298 random design matrix. The probability of a cluster from the original design matrix ( $p$ 299 value) was calculated by the proportion of random design matrices producing a cluster 300 with a cluster value exceeding the original cluster. This test was applied two-sided for 301 negative and positive clusters, which were differentiated by the average slope of the 302 estimated factors. 
303 Monte Carlo cluster tests were performed with 1000 permutations using the test 304 statistics of a repeated measures ANOVA model as the value for clustering (at $\mathrm{p}<.05$ / $305 \mathrm{~F}(1,28)=4.196)$. All tests were performed for low frequencies $(1-30 \mathrm{~Hz})$ and high 306 frequencies (31-100Hz), separately. Muscular and ocular electrodes were excluded from 307 the cluster analysis.

308 The within-subject stimulus intensity factor (which was coded as increasing linearly 309 with stimulus intensity) was tested stimulus-locked from 0 to $1.6 \mathrm{~s}$. The within-subject 310 expectation factor, which was coded as increasing linearly with the cued stimulus 311 intensity was tested cue-locked from 0 to 3.6s. The signed prediction error factor was 312 coded as the difference between stimulus intensity and expectation. The absolute 313 prediction error was coded as the absolute difference between stimulus intensity and 314 expectation (see Figure 2 for details). Additionally, we tested a one-sided prediction 315 error, occurring only when the actual stimulus is of a higher intensity than expected. The 316 signed, absolute and one-sided prediction error factors were tested stimulus-locked 317 from 0 to $1.6 \mathrm{~s}$.

\section{Results}

\section{Behavioral data - aversiveness ratings}

320 Participants experienced aversive heat or saw picture stimuli which were 321 probabilistically cued in terms of modality and intensity, evoking an expectation of 322 modality and intensity. The subsequently applied stimuli were then rated on a visual 323 analog scale (VAS) from 1-4. Our primary behavioral question was whether ratings are 324 influenced by the experimental manipulation of stimulus intensity, expectation and 325 absolute prediction errors.

326 To validate our intensity manipulation for thermal stimuli and to verify the 327 discriminability between different levels of heat, we first tested for the main effect of 328 stimulus intensity (Figure 3a). Our data show a clear rating difference between the three 329 levels of heat. Results regarding the aversive pictures are not the focus of this report but 330 are depicted in Figure $3 \mathrm{~b}$ for the sake of comparison. 

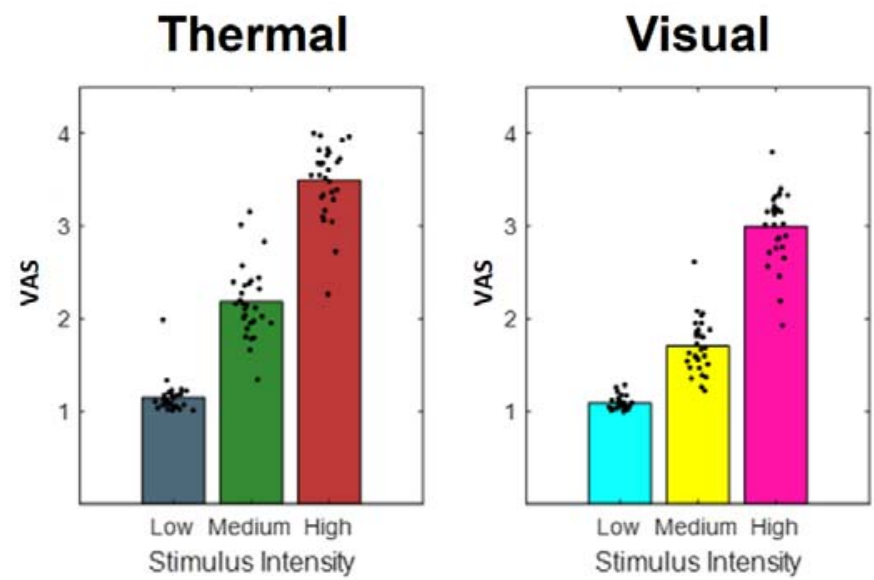

333 Figure 3. Bars indicate pooled aversiveness ratings for a) heat and b) aversive pictures for low, 334 medium and high intensity conditions. Dots indicate average single subject ratings.

335 To evaluate the main effects of stimulus intensity, expectation and absolute prediction 336 errors, we employed a repeated measures ANOVA of the behavioral data, which revealed significant effects for the main effect of stimulus intensity, i.e. the three levels of heat $338(\mathrm{~F}(1,28)=743.97, \mathrm{p}<.001)$. Also, the main effect for expectation on aversiveness ratings 339 was significant $(F(1,28)=38.53, p=<.001)$ (Table 1$)$, indicating an influence of the cued 340 intensity on behavioral aversiveness ratings (Figure 4). The absolute difference between 341 the cued intensity and the actual stimulus intensity (i.e. absolute prediction error), only 342 showed a trend effect on aversiveness ratings $(F(1,28)=2.87, p=.10)$. The results 343 regarding the aversive pictures are summarized in Table 1.

\begin{tabular}{ccccccc}
\hline \multirow{2}{*}{ Factor } & \multicolumn{2}{c}{$\begin{array}{c}\text { Stimulus Intensity } \\
\text { (INT) }\end{array}$} & $\begin{array}{c}\text { Cued Intensity } \\
\text { (EXP) }\end{array}$ & $\begin{array}{c}\text { Absolute } \\
\text { Prediction Error } \\
(\text { PE) }\end{array}$ \\
\cline { 2 - 7 } & $F(1,28)$ & $p$ & $F(1,28)$ & $p$ & $F(1,28)$ & $p$ \\
\hline Modality & & & & & & \\
Thermal & 743.97 & $<.001$ & 39.53 & $<.001$ & 2.87 & .10 \\
Visual & 762.10 & $<.001$ & 1.46 & .24 & 7.7 & .01
\end{tabular}

Table 1. Main effects of stimulus intensity, expectation and absolute prediction errors on 

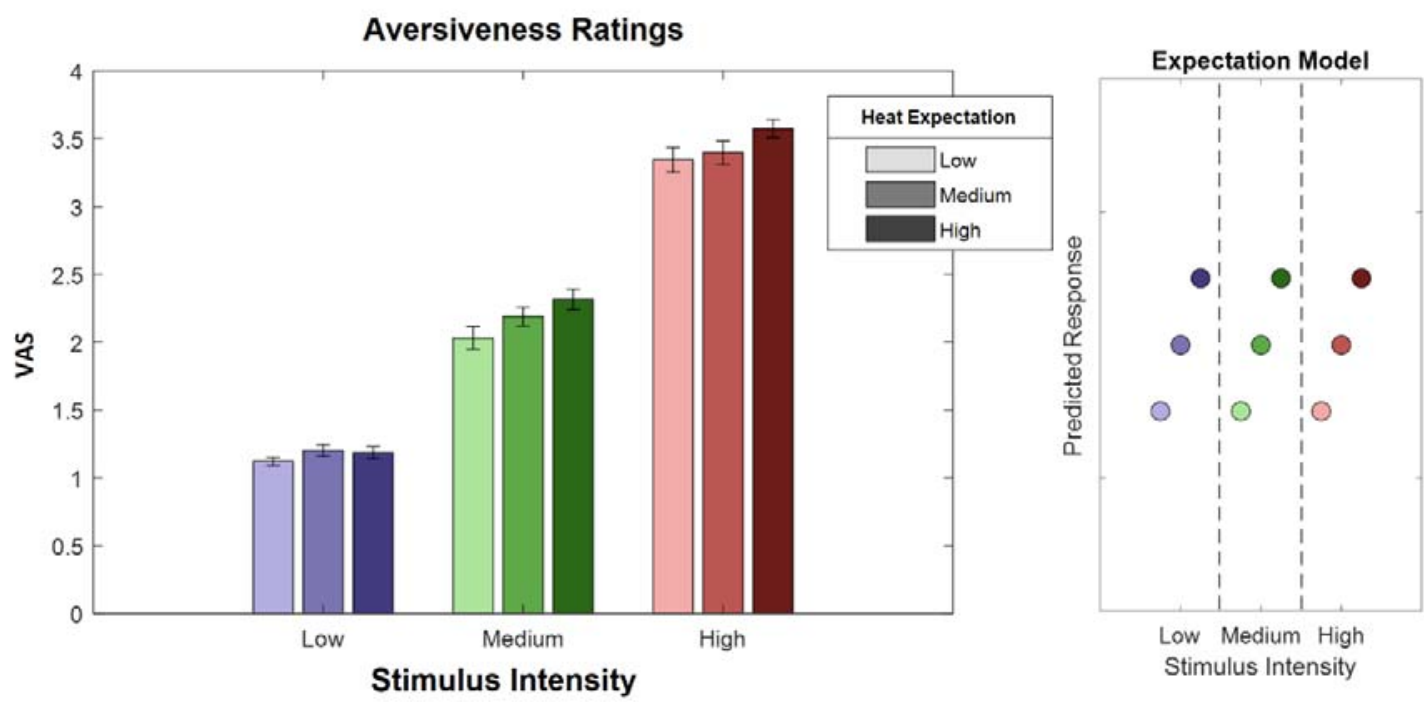

348 Figure 4. Ratings for heat stimuli (left) and "expectation factor" weights (right). Bars indicate average aversiveness ratings. Ratings were given on a scale from 1 to 4 . Error bars depict SEM. The data shows both an effect of stimulus intensity (increase from blue to green to red), but also an effect of expectation (low to medium to high expectation). The right figure represents hypothetical response patterns based on the expectation factor. The $y$-axis represents the hypothetical response variable (e.g. VAS rating). Each dot represents a different condition for each stimulus-cue combination. Blue colors represent low heat conditions, green colors represent medium heat conditions and red colors represent high heat conditions. Color intensities depict expectation level.

\section{EEG - Stimulus Intensity}

358 In a first EEG analysis, we tested for a main effect of the intensity of the heat input in the 359 context of a correctly cued modality (i.e. heat was expected and received). In order to do so, we performed a repeated measures ANOVA on the time-frequency representation of the EEG data on low frequencies $(1-30 \mathrm{~Hz})$ and high frequencies $(31-100 \mathrm{~Hz})$ separately

362 after stimulus onset using a cluster correction criterion to address the multiple 363 comparisons problem (see Methods for details). Any significant cluster - composed of 364 neighboring data points in time, frequency and space - would indicate a neuronal oscillatory representation of variations in stimulus intensity in a given frequency band.

366 In the low frequency range (1-30Hz), one positive cluster (i.e. a positive average slope of 367 the factor) and one negative cluster (i.e. a negative average slope of the factor) were significant (Figure 5), indicating a linear association of stimulus intensity and power in this frequency range. Specifically, the negative cluster included samples in a time range from 250 to $1600 \mathrm{~ms}$ after stimulus onset in a frequency range from 1 to $30 \mathrm{~Hz}$,

371 predominately at contralateral central electrode sites $(p=.002)$. The highest parametric 
372 F-value within this cluster obtained from the repeated measures ANOVA was $F(1,28)=$

$37336.40,(\mathrm{p}<.001)$. This sample was observed at $1250 \mathrm{~ms}$ and $22 \mathrm{~Hz}$ and had a maximum at

374 channel CP2. All channels included samples of the negative low frequency stimulus

375 intensity cluster.

376 Also in the low frequency range $(1-30 \mathrm{~Hz})$, a positive significant cluster included samples

377 in a time range from 150 to $1050 \mathrm{~ms}$ after stimulus onset in the theta frequency range

378 from 1 to $7 \mathrm{~Hz}$ predominately at midline electrode sites ( $\mathrm{p}=.048$ ). The highest

379 parametric F-Value from the repeated measures ANOVA was $F(1,28)=27.93,(p<.001)$.

380 This sample was found at $550 \mathrm{~ms}$ and $3 \mathrm{~Hz}$ and had a maximum at channel 02 . All

381 channels except FC5, CP4, C6 and FT7 were part of the positive low frequency stimulus

382 intensity cluster.

383 In the high frequency range $(31-100 \mathrm{~Hz})$ representing gamma activity one positive 384 cluster was significant $(\mathrm{p}<.001)$. This cluster included samples in a time range from 550

385 to $1600 \mathrm{~ms}$ after stimulus onset and frequencies from 46 to $100 \mathrm{~Hz}$, predominately at 386 contra-lateral centroparietal electrode sites (Figure 5). The highest parametric F-value 387 within this cluster obtained from the repeated measures ANOVA was $F(1,28)=33.35$, ( $p$ $388<.001$ ). This sample was observed at $1600 \mathrm{~ms}$ and $100 \mathrm{~Hz}$ and had a maximum at channel $389 \mathrm{Cz}$. All channels included samples of the positive high frequency stimulus intensity 390 cluster.

391 In conclusion, these results indicate that a higher intensity of the thermal input is 392 associated with increased theta and gamma band power and a negative relationship of 393 alpha-to-beta band power and the intensity of the thermal input (see Figure 5 for a 394 summary of the results of the main effect of stimulus intensity; see Figure 5, figure 395 supplement 1 for single subject differences in the gamma band between low stimulus 396 intensity and high stimulus intensity trials). 


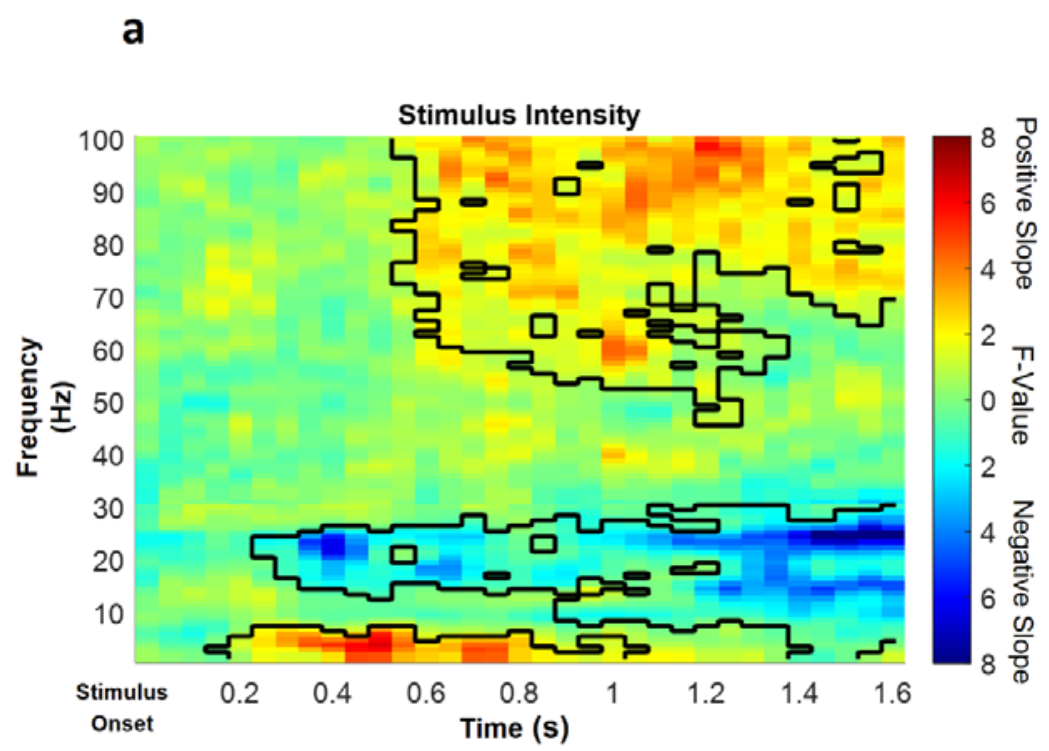

b

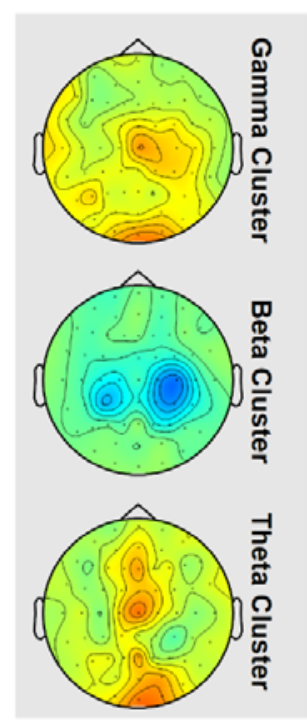

Figure 5. Parametric effects of stimulus intensity. Time-frequency representation averaged 399 over all channels including a significant time-frequency sample of any cluster (a) and 400 topographies over the whole cluster extents (i.e. full time and frequency range), respectively (b) 401 of the stimulus intensity main effect of the repeated measures ANOVA depicting increases 402 (warm) and decreases (cold) in power in relation to heat stimulus intensity. Significant clusters 403 are highlighted. Colors represent F-values from the repeated measures ANOVA statistics for the 404 main effect of stimulus intensity.

\section{Expectation}

406 In a next step, we investigated the representation of the expectation factor in our 407 repeated measures model, again for low frequencies (1-30Hz) and high frequencies (31$408100 \mathrm{~Hz}$ ) separately in the cue-locked time-frequency representation of the EEG data.

409 This analysis revealed one significant positive cluster in the low frequency range (1$41030 \mathrm{~Hz}$ ), indicating a linear association of cue intensity (EXP) and power in this frequency 411 range $(\mathrm{p}<.05)$. The expectation cluster $(\mathrm{p}=.022)$ included samples from time points 412 ranging from $100 \mathrm{~ms}$ to $2000 \mathrm{~ms}$ after cue onset and included frequencies from 1 to 413 20Hz. The highest parametric statistical test value $(\mathrm{F}(1,28)=26.96, \mathrm{p}<.001)$ was 414 observed at channel P1 700ms after cue onset at a frequency of $9 \mathrm{~Hz}$. All channels except 415 TP8 included samples of the late expectation cluster (see Figure 6 for a summary of the 416 results of the expectation cluster; see Figure 6, figure supplement 1 for single subject 417 values). 
418 In summary, these results suggest an increase in alpha-to-beta band power to be 419 associated with our experimental manipulation of expectations regarding the intensity 420 of the thermal input.
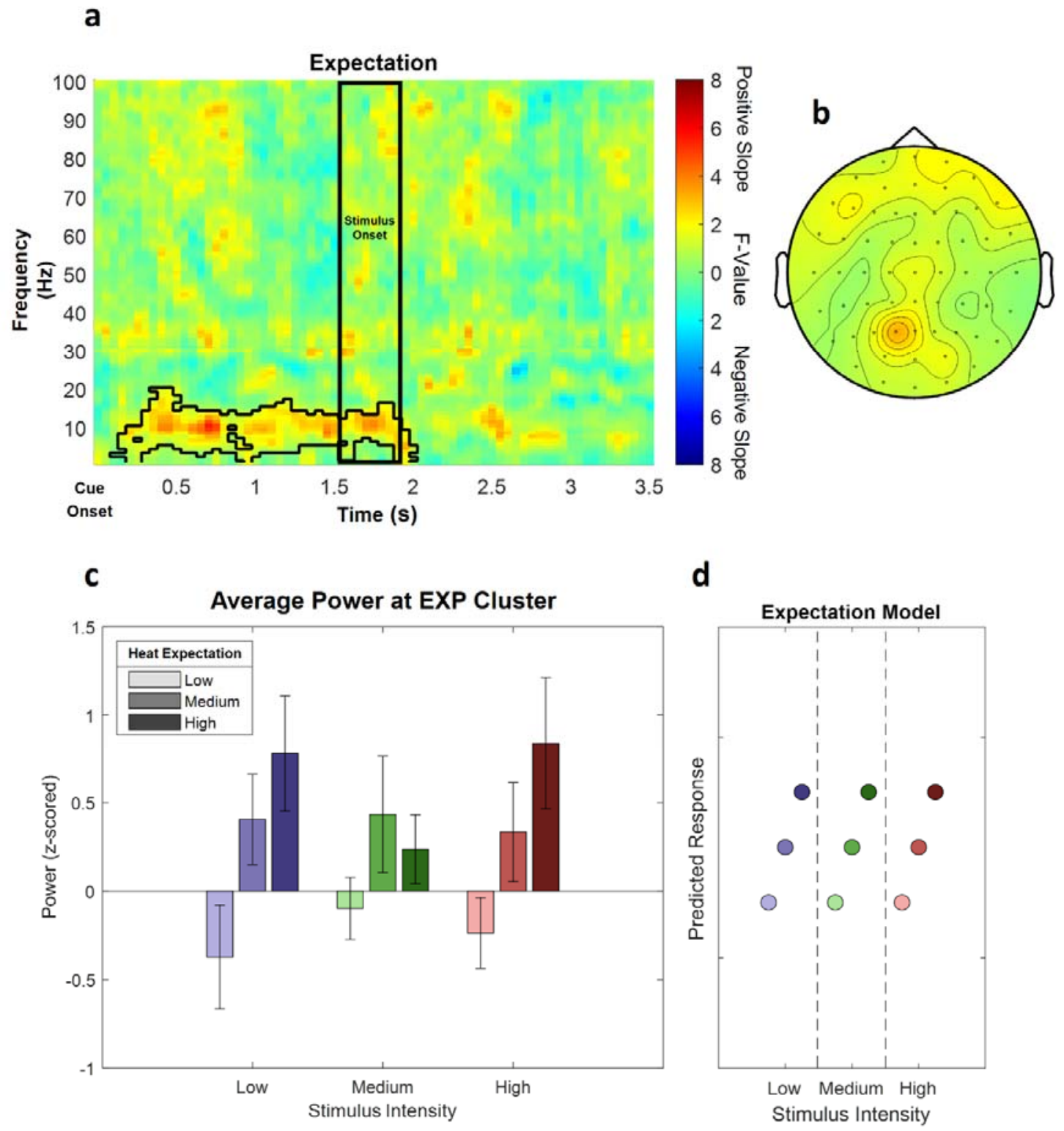

422 Figure 6. The main effect of expectation. a) Time-frequency representation of the statistical F1500 and $1900 \mathrm{~ms}$ marks the jittered onset of the trigger signal to start the ramp up of the heat stimulus. b) Topography of the averaged power over time and frequency of the whole cluster extent (i.e. over the whole time and frequency range) at each channel. Brighter colors indicate higher F-values. c) Power values for all conditions with a valid modality cue (expect heat receive heat) averaged over all significant time-frequency-electrode samples of the EXP cluster show alpha-to-beta enhancement (i.e. positive representation) associated with expectation. Error bars 
430 represent SEM. d) Predicted responses based on the positive expectation factor are shown: The $431 \mathrm{y}$-axis represents an imaginary response variable (e.g. EEG power). Each dot represents a different condition (in the order of the bar plot representation of average EEG power) for each stimulus-cue combination. Blue colors represent low heat conditions, green colors represent medium heat conditions and red colors represent high heat conditions. Color intensities depict expectation level.

\section{Prediction Error Model}

437 Likewise, clustering was performed for the prediction error term after stimulus onset in 438 low $(1-30 \mathrm{~Hz})$ and high frequencies (31-100Hz). Any significant cluster would associate 439 oscillatory activity with the difference of the expectation regarding the intensity of the 440 thermal stimulation and the actual stimulation, representing a violation of this 441 expectation (prediction error).

442 This analysis revealed a significant negative cluster in the high frequency range (31$443100 \mathrm{~Hz}$ ), indicating a (negative) linear association of absolute prediction errors (PE) and 444 power in this frequency range $(\mathrm{p}=.002)$. This (negative) absolute prediction error 445 cluster included samples from frequencies ranging from 51 to $100 \mathrm{~Hz}$ and time points 446 ranging from $50 \mathrm{~ms}$ to $1600 \mathrm{~ms}$ after stimulus onset. The highest parametric statistical 447 test value $(\mathrm{F}(1,28)=28.52, \mathrm{p}<.001)$ was found at channel $011300 \mathrm{~ms}$ after stimulus 448 onset at a frequency of $98 \mathrm{~Hz}$. All channels included samples of the absolute prediction 449 error cluster (see Figure 7 for a summary of the results; see Figure 7, figure supplement 4501 for single subject values).

451 A cluster analysis of the signed prediction error, stimulus-locked after stimulus onset 452 (from $1-30 \mathrm{~Hz}$ for low frequencies and $31-100 \mathrm{~Hz}$ for gamma frequencies; from 0 to $4531600 \mathrm{~ms}$, stimulus-locked) did not reveal any significant cluster of activity associated 454 with a linear increase or decrease of the EXP factor (all $p>.05$ ). Ignoring all stimulus455 cue combinations of the prediction error factor where the stimulus intensity was less 456 intense than expected, leads to a one-sided prediction error factor. A cluster analysis of 457 this effect did not reveal any significant cluster of activity (all $\mathrm{p}>.05$ ).

458 In summary, these results suggest a decrease in gamma band power to be associated 459 with our experimental manipulation of expectation violations, resulting from a 460 mismatch of the cued intensity and the actual heat input. 


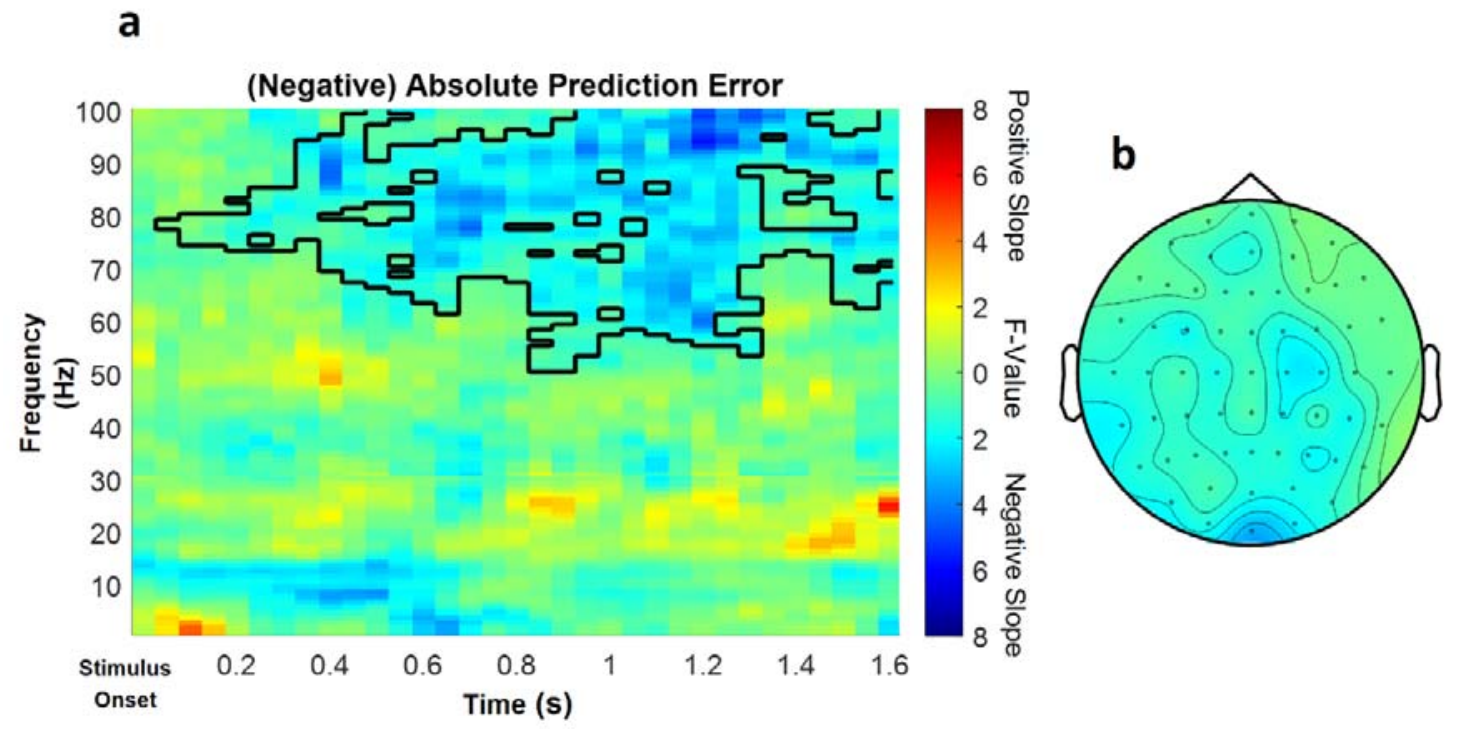

C

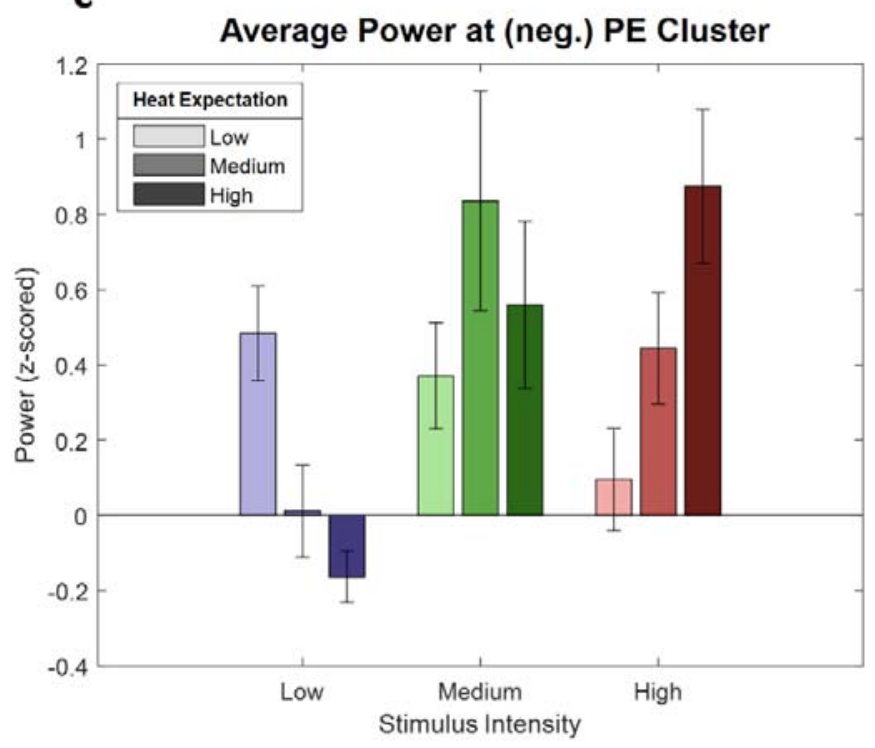

d

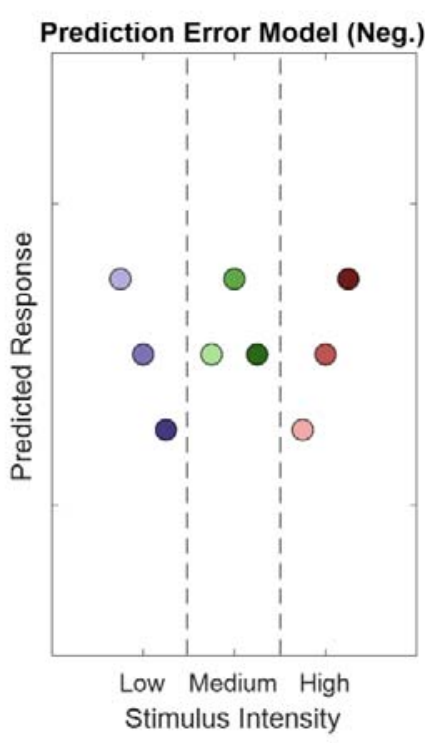

462 Figure 7. The main effect of absolute prediction errors. a) Time-frequency representation of 463 the statistical F-values averaged over all channels. The significant cluster is highlighted. b) 464 Topography of the averaged power over time and frequency of the whole cluster extent (i.e. over 465 the whole time and frequency range) at each channel. Brighter colors indicate higher F-values. c) 466 Power values for all conditions with a valid modality cue (expect heat receive heat) averaged 467 over all significant time-frequency-electrode samples of the PE cluster show gamma decreases 468 (i.e. negative representation) associated with prediction errors. Error bars represent SEM. d) 469 Predicted responses based on the negative prediction error factor are shown: The $y$-axis 470 represents an imaginary response variable (e.g. EEG power). Each dot represents a different 471 condition (in the order of the bar plot representation of average EEG power) for each stimuluscue combination. Blue colors represent low heat conditions, green colors represent medium heat 474 conditions and red colors represent high heat conditions. Color intensities depict expectation level. 


\section{$475 \quad$ Reduced Pain Model}

476 In an additional analysis, we tested all effects in a reduced pain model, which only

477 included painful stimuli (i.e. three expectation levels and two intensity levels).

478 To evaluate the main effects of stimulus intensity, expectation and absolute prediction

479 errors in the behavioral data, we employed again a repeated measures ANOVA which

480 revealed significant effects for the main effect of stimulus intensity, i.e. the two 481 remaining levels of pain $(F(1,28)=1109.9, p<.001)$. Also, the main effect for 482 expectation on pain ratings was significant $(F(1,28)=17.07, p=<.001)$, indicating again 483 an influence of the cued intensity on behavioral pain ratings. The absolute difference 484 between the cued intensity and the actual stimulus intensity (i.e. absolute prediction 485 error) when only painful stimuli were included revealed a positive significant effect on 486 pain ratings $(\mathrm{F}(1,28)=80.75, \mathrm{p}<.001)$. This indicates prediction errors and prior 487 expectations to modulate behavioral aversiveness ratings in painful stimulation.

488 For the analysis of time-frequency EEG data, we performed a repeated measures ANOVA 489 on the time-frequency representation of the EEG data on low frequencies $(1-30 \mathrm{~Hz})$ and 490 high frequencies $(31-100 \mathrm{~Hz})$ separately and again using the same cluster correction 491 criterion to address the multiple comparisons problem as in the initial analysis of the 492 full model.

493 The cluster test of stimulus intensity revealed one negative cluster $(p=.014)$ in the low 494 frequency range $(1-30 \mathrm{~Hz})$ including time points from 850 to $1600 \mathrm{~ms}$ and frequencies 495 from 8 to $30 \mathrm{~Hz}$ (Figure 8a; see Figure 8, figure supplement 1 for single subject values). 496 The maximum statistical F-value $(F(1,28=31.82 ; \mathrm{p}<.001)$ was found at channel AF3 at 497 a frequency of $30 \mathrm{~Hz}$ at $1600 \mathrm{~ms}$ and revealed a similar but more broad topography as 498 compared to the original alpha-to-beta negative main effect of stimulus intensity of the 499 analysis of the full model. All channels included samples of the negative stimulus 500 intensity cluster.

501 In the high frequency range $(31-100 \mathrm{~Hz})$, a negative cluster of activity $(\mathrm{p}=.038)$ was 502 associated with absolute prediction errors and included samples in a time range from 503850 to $1600 \mathrm{~ms}$ after stimulus onset in the gamma frequency range from 54 to $90 \mathrm{~Hz}$ 504 predominately at occipital and parietal electrode sites. The highest parametric F-Value 505 from the repeated measures ANOVA was $F(1,28)=24.10$, $(p<.001)$. This sample was 
506 found at $1150 \mathrm{~ms}$ and $77 \mathrm{~Hz}$ and had a maximum at channel F8 (Figure 8b; see Figure 8, 507 figure supplement 2 for single subject values). All channels except FC5, CP4, C6 and FT7 508 were part of the gamma frequency negative absolute prediction error cluster.

509 In the low $(1-30 \mathrm{~Hz})$ and high frequency $(31-100 \mathrm{~Hz})$ ranges no significant cluster was 510 observed representing a significant relationship between expectations and EEG activity. 511 However, one cluster in the low frequency range $(1-30 \mathrm{~Hz})$ showed a trend level $(\mathrm{p}=$ 5120.14 ; based on cluster mass, i.e. the sum of all clustered F-values) and included samples 513 in a time range from 550 to $1600 \mathrm{~ms}$ after stimulus onset and frequencies from 6 to $24 \mathrm{~Hz}$ 514 and is displayed in dotted lines in Figure 8c (see Figure 8, figure supplement 3 for single 515 subject values). 

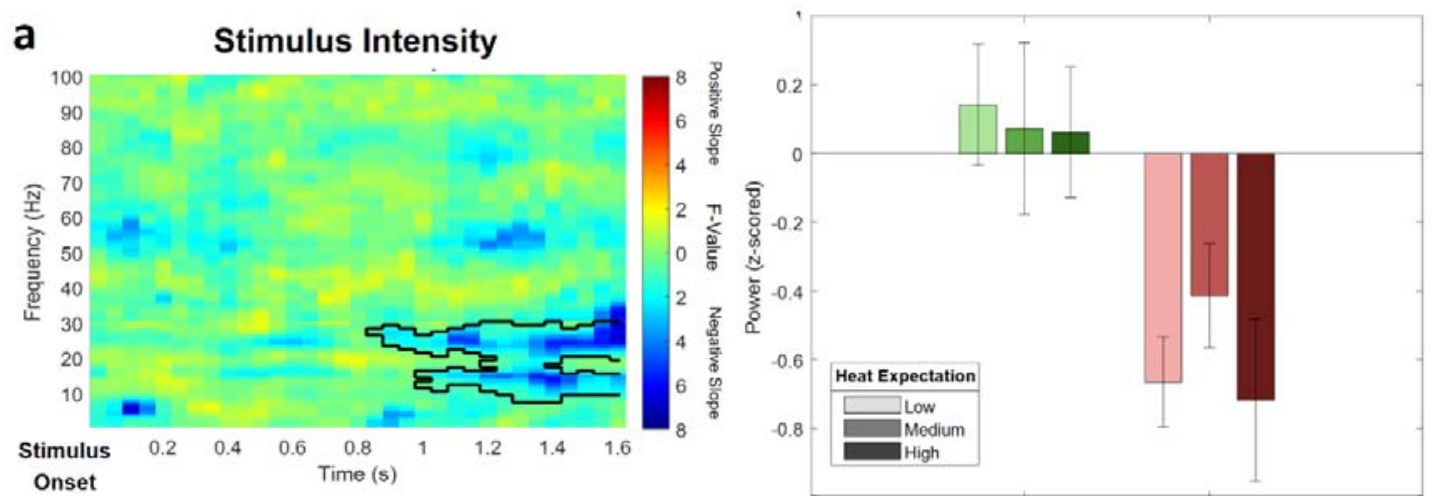

b Neg. Absolute PE
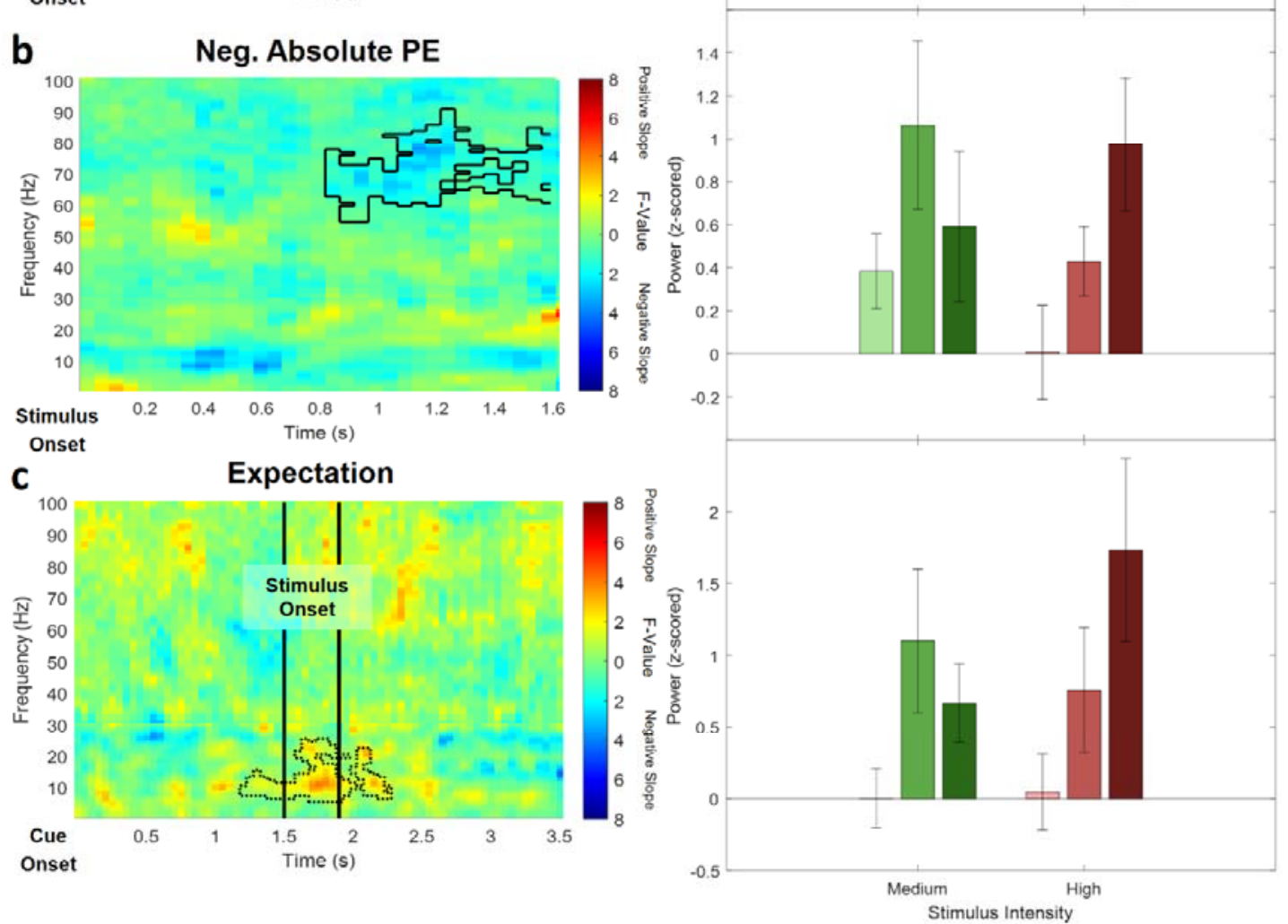

d

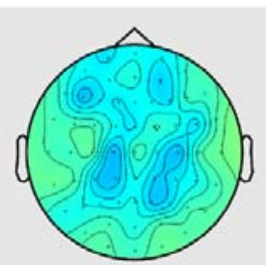

Stimulus Intensity

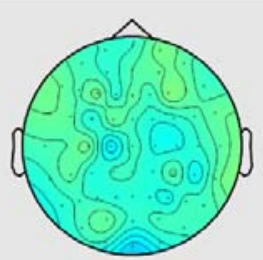

Neg. Abs. PE

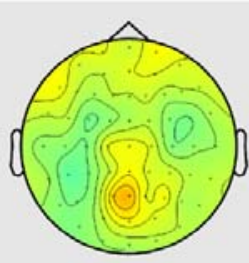

Expectation

Figure 8. EEG data analysis of the reduced pain model. The top three rows show (a) the main effect of stimulus intensity, (b) the main effect of negative absolute prediction errors and (c) the main effect of expectation. Left column: Time-frequency representation of the statistical F-values averaged over all channels. Significant clusters are highlighted by a solid line. The nonsignificant expectation cluster is highlighted by a thin dotted line. Right column: Power values 
523 heat) averaged over all significant time-frequency-electrode samples of the respective cluster. d)

524 Topographies of the averaged power over time and frequency of the whole cluster extent (i.e. 525 over the whole time and frequency range) at each channel for stimulus intensity (left), negative 526 absolute prediction errors (center) and expectation (right), brighter colors indicate higher F527 values.

\section{Discussion}

529 Using a cued heat paradigm with three different stimulus intensities, our data showed a 530 clear discriminability of different levels of aversiveness based on behavioral ratings and 531 EEG time-frequency patterns. Specifically, we observed several clusters of activity to be 532 associated with the intensity of thermal stimulation in the theta, beta and gamma band. 533 Furthermore, behavioral data clearly indicated a positive influence of cued intensity on 534 pain perception. In addition, our results provide evidence for temporally and spectrally 535 separable clusters of oscillatory activity associated with expectation and a negative 536 modulation of gamma activity by prediction errors for thermoception and pain. 537 Specifically, one early low frequency $(1-30 \mathrm{~Hz})$ cluster was related to expectation in 538 thermoception i.e. cued intensity. In contrast, a later occurring cluster at higher 539 frequencies $(31-100 \mathrm{~Hz})$ was related to negative prediction errors in thermoception and 540 pain.

\section{Stimulus intensity and oscillatory activity}

542 Note that our definition of stimulus onset is based on the moment the thermode reached 543 the target temperature. Using a thermode heating gradient of $40^{\circ} \mathrm{C} / \mathrm{s}$ and neglecting any 544 small internal delays, the target temperatures of 42,46 and $48^{\circ} \mathrm{C}$ are reached after 225 , 545325 and $375 \mathrm{~ms}$ respectively. Therefore our observed increase in theta power agrees 546 with previous studies (Ploner et al., 2017) and most likely correspond to pain-related 547 evoked potentials (Lorenz and Garcia-Larrea, 2003; Tiemann et al., 2015), such as the P2 548 with a similar topography. In addition we observed a significant suppression of alpha-to549 beta activity which, given the above mentioned delays of our painful stimuli, is in line 550 with the reported beta suppression in previous EEG studies on pain (Mouraux et al., 551 2003; Ploner et al., 2006; May et al., 2012; Hu et al., 2013). Finally, power in the gamma 552 band was also correlated with heat intensity, which is in line with previous studies 553 (Gross et al., 2007; Hauck et al., 2007; Zhang et al., 2012; Rossiter et al., 2013; Tiemann 554 et al., 2015). Interestingly, only the alpha-to-beta band desynchronization differentiated 555 between medium and high pain conditions, whereas differences in the theta and gamma 
556 band activity were only evident when the lowest stimulus intensity was included which

557 was perceived as neutral.

558 We observed a behavioral effect of prediction errors on perceived stimulus intensity in

559 the reduced pain model, but this effect was only a trend in the full model. The latter

560 finding replicates a previous study (Fazeli and Büchel, 2018) indicating a robust effect.

561 Interestingly, the effect of prediction errors on perception increased, and became

562 significant, when we constrained our analysis to the clearly painful stimuli (reduced

563 pain model). This suggests that a prediction error seems to more strongly affect pain

564 perception whereas the effect is weaker in the context of thermoception. However, this

565 speculation should be corroborated in a future study.

566 On a more conceptual level, the investigation of neurophysiological effects even in the

567 absence of a behavioral effect has been considered meaningful (Wilkinson and Halligan,

568 2004). In particular, the authors argue that because it is commonly unknown which

569 parts of a cognitive process (and in which way) produce a specific behavioral response

570 the relationship between neurophysiological data and behavioral responses should not

571 be overemphasized, and therefore it can be misleading to declare behavioral effects a

572 reference or "gold standard". Studies aiming to understand neurophysiological

573 mechanisms of cognition, usually relate a neurophysiological readout to a known

574 perturbation (i.e. experimental design), which is meaningful in its own right.

\section{Hypotheses based on microcircuits}

576 Theoretical accounts (Arnal and Giraud, 2012; Bastos et al., 2012) have suggested that 577 predictive coding mechanisms could be related to the functional architecture of 578 neuronal microcircuits. As feedforward connections are predominately originating from 579 superficial layers and feedback connections from deep layers, it has been suggested that 580 prediction errors should be expressed by higher frequencies than the predictions that 581 accumulate them.

582 In the auditory modality, these ideas are supported by empirical data (Todorovic et al., 583 2011) showing that prediction errors in the context of repetition suppression were 584 associated with higher gamma band activity. Likewise, in the visual domain, an MEG 585 study has shown that temporo-parietal beta power was correlated with the 
586 predictability of an action kinematics-outcome sequence, while gamma power was

587 correlated with the prediction error (van Pelt et al., 2016).

\section{Frequency Patterns in Predictive Coding of Pain}

589 Only a few studies have investigated the spectral and temporal properties of 590 expectations and prediction errors in the context of pain (summarized by Ploner et al., 591 2017). A recent study in rodents has suggested an information flow between S1 gamma 592 and ACC beta activity during spontaneous pain (Xiao et al., 2019). Based on these data, 593 the authors have proposed a predictive coding model including a bottom-up (gamma) 594 and top-down (beta) component (Song et al., 2019). Finally, in humans a recent EEG 595 study showed that the sensorimotor cortex is more strongly connected to the medial 596 prefrontal cortex at alpha frequencies during tonic pain, suggesting alpha band activity 597 in tonic pain to be associated with bottom-up instead of top-down signaling (Nickel et 598 al., 2020). Nevertheless, the focus of these studies was on generic interactions (i.e. top 599 down versus bottom up) processes without directly inducing prediction errors as in a 600 cued pain paradigm employed in our study.

601 In the flexible routing model proposed by Ploner et al. (2017), pain is seen as driven by 602 contextual processes, such as expectations, which is associated with alpha/beta 603 oscillations and alpha/beta synchrony across brain areas. Previous studies have started 604 to examine the spectral properties of mechanisms related to generative models of pain 605 perception. In particular, a previous MEG study reported that alpha suppression in the 606 anterior insula is related mainly to pain expectation in a paradigm in which painful 607 stimuli were interleaved with non-painful stimuli (Franciotti et al., 2009). This was 608 interpreted as a preparatory mechanism for an upcoming painful stimulus. In a related 609 study, alpha desynchronization in the context of predictable painful stimuli, has been 610 discussed as a possible neural correlate of attentional preparatory processes (Babiloni 611 et al., 2003).

612 Expectation is also a crucial ingredient of placebo analgesia and nocebo hyperalgesia. A 613 previous study reported that resting state alpha band activity was also linked to the 614 expectation of pain modulation (analgesia) in a placebo paradigm (Huneke et al., 2013). 615 With respect to negative expectations, it has been shown that pain modulation due to 616 nocebo expectation is associated with enhanced alpha activity (Albu and Meagher, 
617 2016). Our findings are in line with these results indicating an important role of low 618 frequency activity in mediating expectation effects in a pain network underlying a 619 generative model for pain perception.

620 In contrast to prediction error effects in the visual (Bauer et al., 2014; van Pelt et al., 621 2016) and auditory (Edwards et al., 2005; Parras et al., 2017) domains, we observed a 622 negative modulation of gamma activity by absolute prediction errors. However it should 623 be noted that opposite effects have been observed in other cognitive domains. For 624 instance, increased gamma power has been associated with successful matching (i.e. the 625 absence of a prediction error) between external input and internal representation 626 (Herrmann et al., 2004a; Osipova et al., 2006; Wang et al., 2018). In particular, gamma627 band responses have been explained in terms of the match between bottom-up and top628 down information (Herrmann et al., 2004b). One example is the observation of 629 increased gamma activity with a higher so called cloze probability in sentence-level 630 language comprehension (Hald et al., 2006; Obleser and Kotz, 2011; Wang et al., 2012, 631 2018; Molinaro et al., 2013). It has been shown that a critical word that is semantically 632 predictable by the preceding sentence (so-called high cloze probability) induces a larger 633 gamma response than words which are semantically incongruent (i.e. unpredicted; low 634 cloze probability) (Wang et al., 2018).

\section{Pain versus thermoception}

636 In the present study, the lowest stimulus intensity was often not perceived as painful but 637 as hot. In general, stimulus properties were chosen to be comparable to a previous fMRI 638 study which showed fMRI signals related to prediction errors (Fazeli and Büchel, 2018). 639 However, even though the lowest stimulus intensity $\left(42^{\circ} \mathrm{C}\right)$ was above the threshold of 640 nociceptors (Treede et al., 1998), the subjective experience of the lowest pain stimuli 641 was often rated as neutral. Therefore, we performed an additional analysis (reduced 642 pain model) only comprising clearly painful stimuli $\left(46^{\circ} \mathrm{C}\right.$ and $\left.48^{\circ} \mathrm{C}\right)$ to more specifically 643 address expectations and predictions errors in pain. The analyses of the behavioral data 644 revealed similar results. Both models showed a highly significant effect of stimulus 645 intensity and expectation on perceived stimulus intensity. In addition, the reduced pain 646 model showed a significant prediction error effect, which was formally not observed in 647 the full model. However, it is important to note that this difference should not be over 648 interpreted as the p-value for the prediction error effect of the full model was at a trend 
649 level $(\mathrm{p}=0.1)$. Importantly, the negative representation of prediction errors in the 650 gamma band was evident in both, the reduced and the full model.

\section{Limitations}

652 To unravel, the temporal aspects of expectations and prediction errors, this study has 653 been designed in close analogy to a previous fMRI study and we decided to use the same 654 experimental paradigm (Fazeli and Büchel, 2018). We therefore decided to also keep the 655 sample characteristics similar and restricted the sample to male participants, which

656 means that we cannot generalize our results to the population. However, our study 657 agrees with findings of a previous study using a similar design (Geuter et al., 2017) 658 which tested male and female participants. Future studies should investigate samples 659 including female participants. This would also allow to investigate sex effects with 660 respect to expectation and prediction error effects in pain.

661 To minimize motor responses and speed-up the rating procedure, we used a 4-button 662 device to directly assess stimulus intensity (in contrast to using two buttons to move a 663 slider on a VAS), thus being limited to a coarse rating scale of 4 levels, where 1 was 664 labeled as "neutral" and 4 was labeled as "very strong". This allows to accommodate 665 more trials, but is not ideal to assess fine-grained differences, specifically to differentiate 666 between non-painful and painful stimulation, as level 1 would represent 0-25 on a 0-100 667 VAS. Future research could use conventional 0-100 VAS to assess stimulus intensity on a 668 finer scale.

669 For reasons of comparability to a previous fMRI study, we employed three different 670 temperatures for all volunteers. Alternatively, we could have defined three levels of pain 671 based on individual calibration of heat stimuli (Taesler and Rose, 2017; Grahl et al., 672 2018; Horing et al., 2019; Zhang et al., 2020; Feldhaus et al., 2021). Such a procedure 673 could have avoided trials where no pain was subjectively perceived. On the other hand, 674 such an approach also carries the risk that subjective ratings during the calibration 675 process do not truly reflect pain and can lead to errors (especially if ratings are too low) 676 which then affect the entire experiment. However, to address this shortcomming, we 677 performed an additional analysis, which only included painful stimulus intensities. 


\section{Summary}

679 Our data show that key variables required for pain perception and thermoception in the 680 context of a generative model are correlated with distinct oscillatory profiles in the 681 brain. Furthermore, each oscillatory frequency band was correlated with a distinct 682 variable such as expectation and prediction errors. These mechanistic insights could be 683 very helpful in patients with acute and more importantly in patients with chronic pain, 684 where expectations have been shown to play a critical role in pain persistence.

\section{Acknowledgements}

686 We would like to thank Markus Ploner for comments on an earlier version of this 687 manuscript. We would also like to thank Matthias Kerkemeyer for his help during data 688 collection. C.B is supported by the DFG, SFB 289, M.R. is supported by the DFG SFB TR 689169 project B3.

690 


\section{References}

692 Albu S, Meagher MW (2016) Expectation of nocebo hyperalgesia affects EEG alpha-

693 activity. Int J Psychophysiol 109:147-152.

694 Anchisi D, Zanon M (2015) A Bayesian perspective on sensory and cognitive integration 695 in pain perception and placebo analgesia. PLoS ONE 10:e0117270.

Arnal LH, Giraud A-L (2012) Cortical oscillations and sensory predictions. Trends Cogn Sci (Regul Ed) 16:390-398.

698

Atlas LY, Bolger N, Lindquist MA, Wager TD (2010) Brain Mediators of Predictive Cue Effects on Perceived Pain. Journal of Neuroscience 30:12964-12977.

Atlas LY, Wager TD (2012) How expectations shape pain. Neurosci Lett 520:140-148.

701

702

703

704

705

706

707

708

709

710

711

712

713

714

715

716

717

718

719

720

721

722

723

724

Babiloni C, Brancucci A, Babiloni F, Capotosto P, Carducci F, Cincotti F, Arendt-Nielsen L, Chen ACN, Rossini PM (2003) Anticipatory cortical responses during the expectancy of a predictable painful stimulation. A high-resolution electroencephalography study. Eur J Neurosci 18:1692-1700.

Bastos AM, Usrey WM, Adams RA, Mangun GR, Fries P, Friston KJ (2012) Canonical microcircuits for predictive coding. Neuron 76:695-711.

Bauer M, Oostenveld R, Peeters M, Fries P (2006) Tactile spatial attention enhances gamma-band activity in somatosensory cortex and reduces low-frequency activity in parieto-occipital areas. J Neurosci 26:490-501.

Bauer M, Stenner M-P, Friston KJ, Dolan RJ (2014) Attentional Modulation of Alpha/Beta and Gamma Oscillations Reflect Functionally Distinct Processes. Journal of Neuroscience 34:16117-16125.

Bingel U, Lorenz J, Schoell E, Weiller C, Büchel C (2006) Mechanisms of placebo analgesia: rACC recruitment of a subcortical antinociceptive network. Pain 120:8-15.

Büchel C, Geuter S, Sprenger C, Eippert F (2014) Placebo Analgesia: A Predictive Coding Perspective. Neuron 81:1223-1239.

Clark A (2013) Whatever next? Predictive brains, situated agents, and the future of cognitive science. Behav Brain Sci 36:181-204.

Colloca L, Benedetti F (2005) Placebos and painkillers: is mind as real as matter? Nat Rev Neurosci 6:545-552.

Debener S, Herrmann CS, Kranczioch C, Gembris D, Engel AK (2003) Top-down attentional processing enhances auditory evoked gamma band activity. Neuroreport 14:683-686. 
725 Edwards E, Soltani M, Deouell LY, Berger MS, Knight RT (2005) High Gamma Activity in

726

727

728

729

730

731

732

733

734

735

736

737

738

739

740

741

742

743

744

745

746

747

748

749

750

751

752

753

754

755

756

757

758

759

760

761

Response to Deviant Auditory Stimuli Recorded Directly From Human Cortex. Journal of Neurophysiology 94:4269-4280.

Egner T, Monti JM, Summerfield C (2010) Expectation and Surprise Determine Neural Population Responses in the Ventral Visual Stream. J Neurosci 30:16601-16608.

Ernst MO, Banks MS (2002) Humans integrate visual and haptic information in a statistically optimal fashion. Nature 415:429-433.

Fazeli S, Büchel C (2018) Pain-Related Expectation and Prediction Error Signals in the Anterior Insula Are Not Related to Aversiveness. J Neurosci 38:6461-6474.

Feldhaus MH, Horing B, Sprenger C, Büchel C (2021) Association of nocebo hyperalgesia and basic somatosensory characteristics in a large cohort. Scientific Reports $11: 762$.

Franciotti R, Ciancetta L, Della Penna S, Belardinelli P, Pizzella V, Romani GL (2009) Modulation of alpha oscillations in insular cortex reflects the threat of painful stimuli. Neuroimage 46:1082-1090.

Friston K (2010) The free-energy principle: a unified brain theory? Nat Rev Neurosci 11:127-138.

Geuter S, Boll S, Eippert F, Büchel C (2017) Functional dissociation of stimulus intensity encoding and predictive coding of pain in the insula. Elife 6.

Grahl A, Onat S, Büchel C (2018) The periaqueductal gray and Bayesian integration in placebo analgesia. Elife 7.

Grandchamp R, Delorme A (2011) Single-Trial Normalization for Event-Related Spectral 912 Decomposition Reduces Sensitivity to Noisy Trials. Front Psychol 2.

Gross J, Schnitzler A, Timmermann L, Ploner M (2007) Gamma Oscillations in Human Primary Somatosensory Cortex Reflect Pain Perception Fries P, ed. PLoS Biol 5:e133.

Gruber T, Müller MM, Keil A, Elbert T (1999) Selective visual-spatial attention alters induced gamma band responses in the human EEG. Clin Neurophysiol 110:20742085.

Hald LA, Bastiaansen MCM, Hagoort P (2006) EEG theta and gamma responses to semantic violations in online sentence processing. Brain and Language 96:90105.

Hauck M, Lorenz J, Domnick C, Gerloff C, Engel AK (2015) Top-Down and Bottom-Up Modulation of Pain-Induced Oscillations. Front Hum Neurosci 9

Hauck M, Lorenz J, Engel AK (2007) Attention to Painful Stimulation Enhances $\gamma$-Band Activity and Synchronization in Human Sensorimotor Cortex. The Journal of Neuroscience 27:9270. 
762 Herrmann C, Lenz D, Junge S, Busch N, Maess B (2004a) Memory-matches evoke human 763 gamma-responses. BMC neuroscience 5:13.

764 Herrmann CS, Munk MHJ, Engel AK (2004b) Cognitive functions of gamma-band activity: 765 memory match and utilization. Trends in Cognitive Sciences 8:347-355.

766 Horing B, Sprenger C, Büchel C (2019) The parietal operculum preferentially encodes 767 heat pain and not salience. PLOS Biology 17:e3000205.

$768 \mathrm{Hu}$ L, Peng W, Valentini E, Zhang Z, Hu Y (2013) Functional features of nociceptiveinduced suppression of alpha band electroencephalographic oscillations. J Pain 14:89-99.

771

772

773

774

775

776

777

778

779

780

781

782

783

784

785

786

787

788

789

790

791

792

793

794

795

796

797

Huang Y, Rao RPN (2011) Predictive coding. Wiley Interdisciplinary Reviews-Cognitive Science 2:580-593.

Huneke NTM, Brown CA, Burford E, Watson A, Trujillo-Barreto NJ, El-Deredy W, Jones AKP (2013) Experimental placebo analgesia changes resting-state alpha oscillations. PLOS ONE 8:e78278.

Jung TP, Makeig S, Humphries C, Lee TW, McKeown MJ, Iragui V, Sejnowski TJ (2000) Removing electroencephalographic artifacts by blind source separation. Psychophysiology 37:163-178.

Klem GH, Lüders HO, Jasper HH, Elger C (1999) The ten-twenty electrode system of the International Federation. The International Federation of Clinical Neurophysiology. Electroencephalogr Clin Neurophysiol Suppl 52:3-6.

Knill DC, Pouget A (2004) The Bayesian brain: the role of uncertainty in neural coding and computation. Trends Neurosci 27:712-719.

Koyama T, McHaffie JG, Laurienti PJ, Coghill RC (2005) The subjective experience of pain: where expectations become reality. Proc Natl Acad Sci USA 102:1295012955.

Lang, P.J., Bradley, M.M., \& Cuthbert, B.N. (2008). International affective picture system (IAPS): Affective ratings of pictures and instruction manual. Technical Report A8. University of Florida, Gainesville, FL.

Liberati G, Klöcker A, Algoet M, Mulders D, Maia Safronova M, Ferrao Santos S, Ribeiro Vaz J-G, Raftopoulos C, Mouraux A (2018) Gamma-Band Oscillations Preferential for Nociception can be Recorded in the Human Insula. Cereb Cortex 28:36503664 .

Lorenz J, Garcia-Larrea L (2003) Contribution of attentional and cognitive factors to laser evoked brain potentials. Neurophysiol Clin 33:293-301.

Makeig S, Bell A, Jung T-P, Sejnowski T (1996) Independent Component Analysis of Electroencephalographic Data. 8. 
798

799

800

801

802

803

804

805

806

807

808

809

810

811

812

813

814

815

816

817

818

819

820

821

822

823

824

825

826

827

828

829

830

831

832

833

834

835
May ES, Butz M, Kahlbrock N, Hoogenboom N, Brenner M, Schnitzler A (2012) Pre- and post-stimulus alpha activity shows differential modulation with spatial attention during the processing of pain. Neuroimage 62:1965-1974.

Molinaro N, Barraza P, Carreiras M (2013) Long-range neural synchronization supports fast and efficient reading: EEG correlates of processing expected words in sentences. NeuroImage 72:120-132.

Mouraux A, Guérit JM, Plaghki L (2003) Non-phase locked electroencephalogram (EEG) responses to $\mathrm{CO} 2$ laser skin stimulations may reflect central interactions between A partial partial differential- and C-fibre afferent volleys. Clin Neurophysiol 114:710-722.

Nickel MM, Dinh ST, May ES, Tiemann L, Hohn VD, Gross J, Ploner M (2020) Neural oscillations and connectivity characterizing the state of tonic experimental pain in humans. Human Brain Mapping 41:17-29.

Obleser J, Kotz SA (2011) Multiple brain signatures of integration in the comprehension of degraded speech. NeuroImage 55:713-723.

Ongaro G, Kaptchuk TJ (2019) Symptom perception, placebo effects, and the Bayesian brain. Pain 160:1-4.

Oostenveld R, Fries P, Maris E, Schoffelen J-M (2011) FieldTrip: Open Source Software for Advanced Analysis of MEG, EEG, and Invasive Electrophysiological Data. Computational intelligence and neuroscience 2011:156869.

Osipova D, Takashima A, Oostenveld R, Fernández G, Maris E, Jensen 0 (2006) Theta and Gamma Oscillations Predict Encoding and Retrieval of Declarative Memory. J Neurosci 26:7523-7531.

Parras GG, Nieto-Diego J, Carbajal GV, Valdés-Baizabal C, Escera C, Malmierca MS (2017) Neurons along the auditory pathway exhibit a hierarchical organization of prediction error. Nature Communications 8:2148.

Petrovic P, Kalso E, Petersson KM, Ingvar M (2002) Placebo and opioid analgesia-imaging a shared neuronal network. Science 295:1737-1740.

Ploghaus A, Tracey I, Gati JS, Clare S, Menon RS, Matthews PM, Rawlins JN (1999) Dissociating pain from its anticipation in the human brain. Science 284:19791981.

Ploner M, Gross J, Timmermann L, Pollok B, Schnitzler A (2006) Pain suppresses spontaneous brain rhythms. Cereb Cortex 16:537-540.

Ploner M, Sorg C, Gross J (2017) Brain Rhythms of Pain. Trends Cogn Sci (Regul Ed) 21:100-110.

Rossiter HE, Worthen SF, Witton C, Hall SD, Furlong PL (2013) Gamma oscillatory amplitude encodes stimulus intensity in primary somatosensory cortex. Front Hum Neurosci

7

Available

at: 
836

837

838

839

840

841

842

843

844

845

846

847

848

849

850

851

852

853

854

855

856

857

858

859

860

861

862

863

864

865

866

867

868

869

870

871

872

https://www.frontiersin.org/articles/10.3389/fnhum.2013.00362/full

[Accessed December 2, 2020].

Schulz E, May ES, Postorino M, Tiemann L, Nickel MM, Witkovsky V, Schmidt P, Gross J, Ploner M (2015) Prefrontal Gamma Oscillations Encode Tonic Pain in Humans. Cereb Cortex 25:4407-4414.

Song Y, Yao M, Kemprecos H, Byrne Á, Xiao Z, Zhang Q, Singh A, Wang J, Chen ZS (2019) Predictive Coding Models for Pain Perception. Neuroscience. Available at: http://biorxiv.org/lookup/doi/10.1101/843284 [Accessed March 31, 2020].

Summerfield C, de Lange FP (2014) Expectation in perceptual decision making: neural and computational mechanisms. Nature Reviews Neuroscience 15:745-756.

Taesler P, Rose M (2016) Prestimulus Theta Oscillations and Connectivity Modulate Pain Perception. J Neurosci 36:5026-5033.

Taesler P, Rose M (2017) Psychophysically-anchored, Robust Thresholding in Studying Pain-related Lateralization of Oscillatory Prestimulus Activity. Journal of Visualized Experiments 2017.

Tallon-Baudry C, Kreiter A, Bertrand O (1999) Sustained and transient oscillatory responses in the gamma and beta bands in a visual short-term memory task in humans. Vis Neurosci 16:449-459.

Tiemann L, May ES, Postorino M, Schulz E, Nickel MM, Bingel U, Ploner M (2015) Differential neurophysiological correlates of bottom-up and top-down modulations of pain. Pain 156:289-296.

Tiemann L, Schulz E, Gross J, Ploner M (2010) Gamma oscillations as a neuronal correlate of the attentional effects of pain. Pain 150:302-308.

Tiitinen H, Sinkkonen J, Reinikainen K, Alho K, Lavikainen J, Näätänen R (1993) Selective attention enhances the auditory $40-\mathrm{Hz}$ transient response in humans. Nature 364:59-60.

Todorovic A, van Ede F, Maris E, de lange FP (2011) Prior Expectation Mediates Neural Adaptation to Repeated Sounds in the Auditory Cortex: An MEG Study. Journal of Neuroscience 31:9118-9123.

Tracey I, Mantyh PW (2007) The cerebral signature for pain perception and its modulation. Neuron 55:377-391.

Treede RD, Meyer RA, Campbell JN (1998) Myelinated mechanically insensitive afferents from monkey hairy skin: heat-response properties. J Neurophysiol 80:10821093.

Tu Y, Zhang Z, Tan A, Peng W, Hung YS, Moayedi M, Iannetti GD, Hu L (2016) Alpha and gamma oscillation amplitudes synergistically predict the perception of forthcoming nociceptive stimuli. Human Brain Mapping 37:501-514. 
873

874

875

876

877

878

879

880

881

882

883

884

885

886

887

888

889

890

891

892

893

894

895

896

897

898

899

900

901

van Pelt S, Heil L, Kwisthout J, Ondobaka S, van Rooij I, Bekkering H (2016) Beta- and gamma-band activity reflect predictive coding in the processing of causal events. Soc Cogn Affect Neurosci 11:973-980.

Wager TD, Rilling JK, Smith EE, Sokolik A, Casey KL, Davidson RJ, Kosslyn SM, Rose RM, Cohen JD (2004) Placebo-induced changes in FMRI in the anticipation and experience of pain. Science 303:1162-1167.

Wang L, Hagoort P, Jensen $O$ (2018) Gamma Oscillatory Activity Related to Language Prediction. Journal of Cognitive Neuroscience 30:1075-1085.

Wang L, Zhu Z, Bastiaansen M (2012) Integration or Predictability? A Further Specification of the Functional Role of Gamma Oscillations in Language Comprehension. Front Psychol 3 Available at: https://www.frontiersin.org/articles/10.3389/fpsyg.2012.00187/full [Accessed February 2, 2021].

Wiech K (2016) Deconstructing the sensation of pain: The influence of cognitive processes on pain perception. Science 354:584-587.

Wiech K, Ploner M, Tracey I (2008) Neurocognitive aspects of pain perception. Trends Cogn Sci (Regul Ed) 12:306-313.

Wilkinson D, Halligan P (2004) The relevance of behavioural measures for functionalimaging studies of cognition. Nature Reviews Neuroscience 5:67-73.

Xiao Z, Martinez E, Kulkarni PM, Zhang Q, Hou Q, Rosenberg D, Talay R, Shalot L, Zhou H, Wang J, Chen ZS (2019) Cortical Pain Processing in the Rat Anterior Cingulate Cortex and Primary Somatosensory Cortex. Front Cell Neurosci 13:165.

Zhang S, Yoshida W, Mano H, Yanagisawa T, Mancini F, Shibata K, Kawato M, Seymour B (2020) Pain Control by Co-adaptive Learning in a Brain-Machine Interface. Current Biology 30:3935-3944.e7.

Zhang ZG, Hu L, Hung YS, Mouraux A, Iannetti GD (2012) Gamma-band oscillations in the primary somatosensory cortex--a direct and obligatory correlate of subjective pain intensity. J Neurosci 32:7429-7438. 
902

\section{Supplementary Materials}

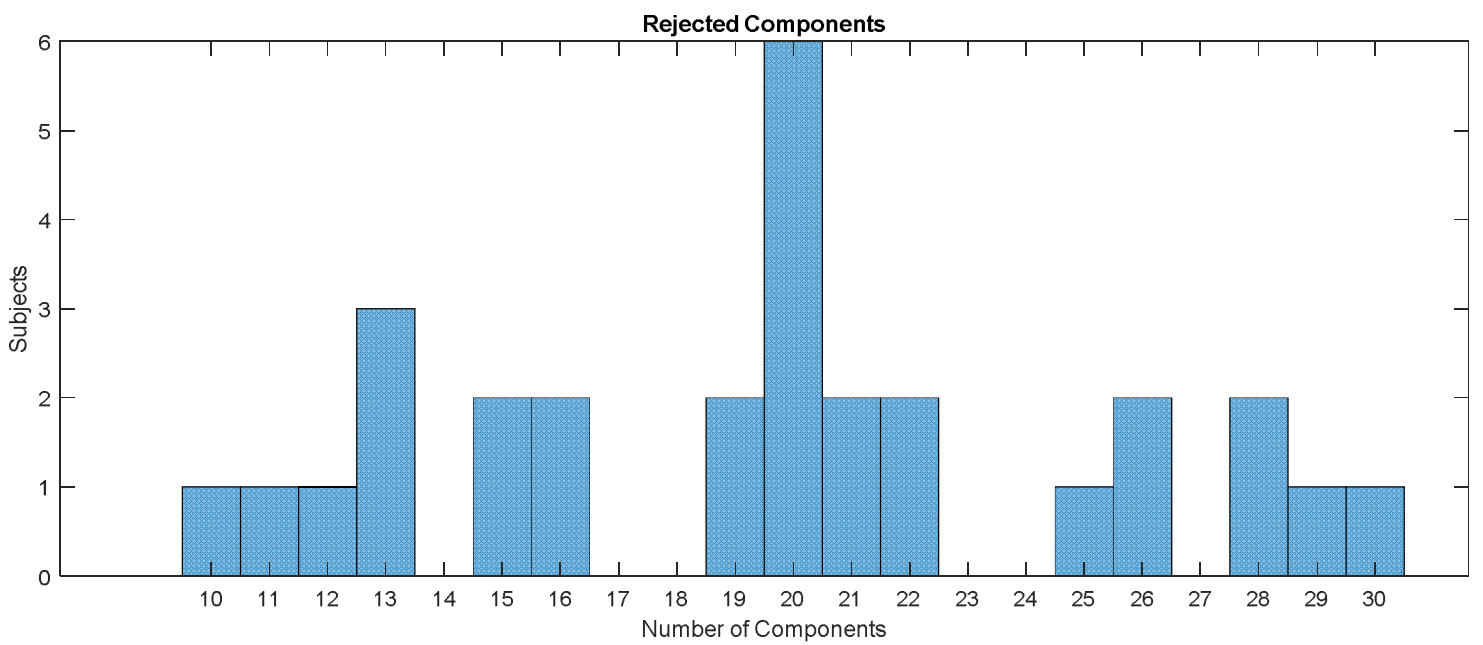

905 Figure 1-figure supplement 1. Histogram showing the distribution of the total number of rejected components based on detected muscle artifacts. 
bioRxiv preprint doi: https://doi.org/10.1101/2020.09.10.291658; this version posted February 5, 2021. The copyright holder for this preprint (which was not certified by peer review) is the author/funder, who has granted bioRxiv a license to display the preprint in perpetuity. It is made available under aCC-BY 4.0 International license.

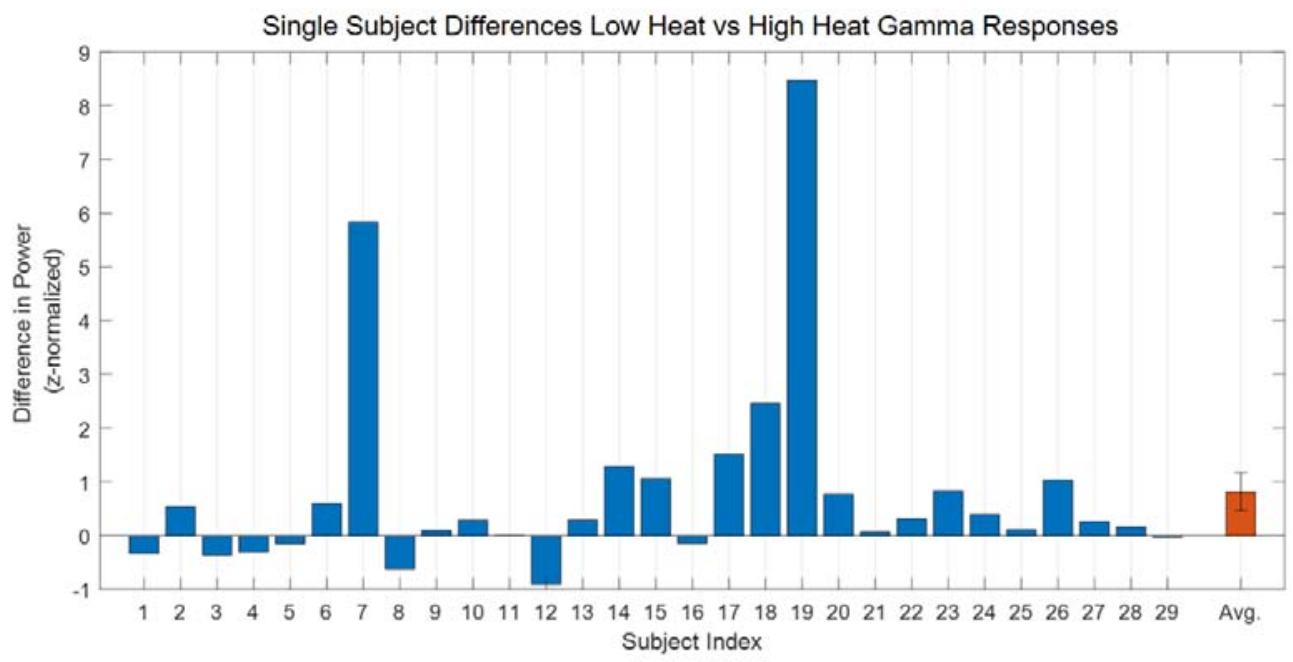

911

912 Figure 5-figure supplement 1. Difference for the main effect of stimulus intensity in the 913 gamma band (averaged over $60-100 \mathrm{~Hz}, 1250$ to $1600 \mathrm{~ms}$ ) in power values for all high heat vs. 914 low heat conditions with a valid modality cue (expect heat receive heat) for each subject, 915 respectively. 


\section{Expectation}
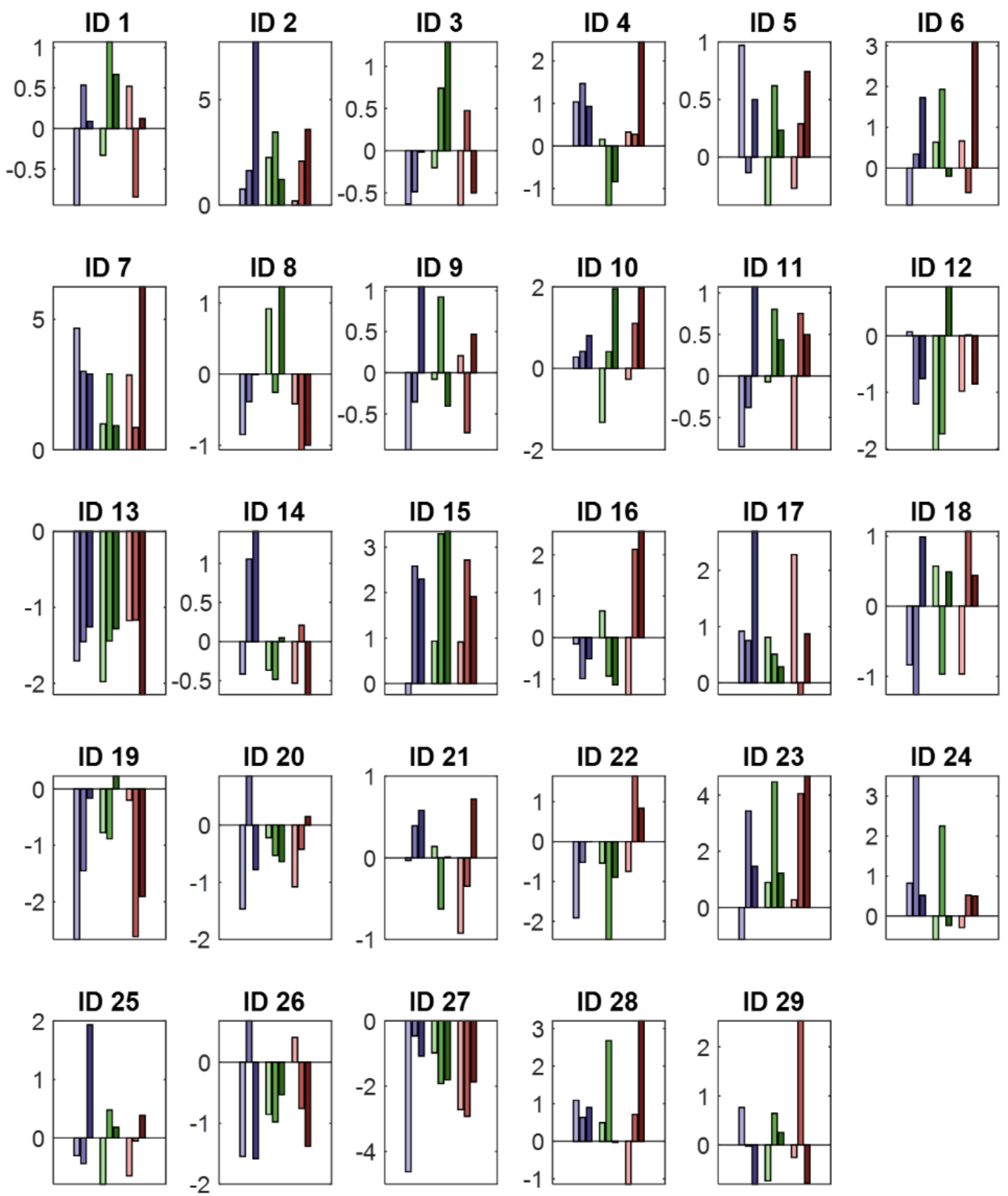

918 Figure 6-figure supplement 1. Power values for all conditions with a valid modality cue

919 (expect heat receive heat) averaged over all significant time-frequency-electrode samples period

920 for each subject (ID) of the EXP cluster. Blue colors represent low heat conditions, green colors

921 represent medium heat conditions and red colors represent high heat conditions. Color intensities depict expectation level. 


\section{Neg. Absolute Prediction Error}
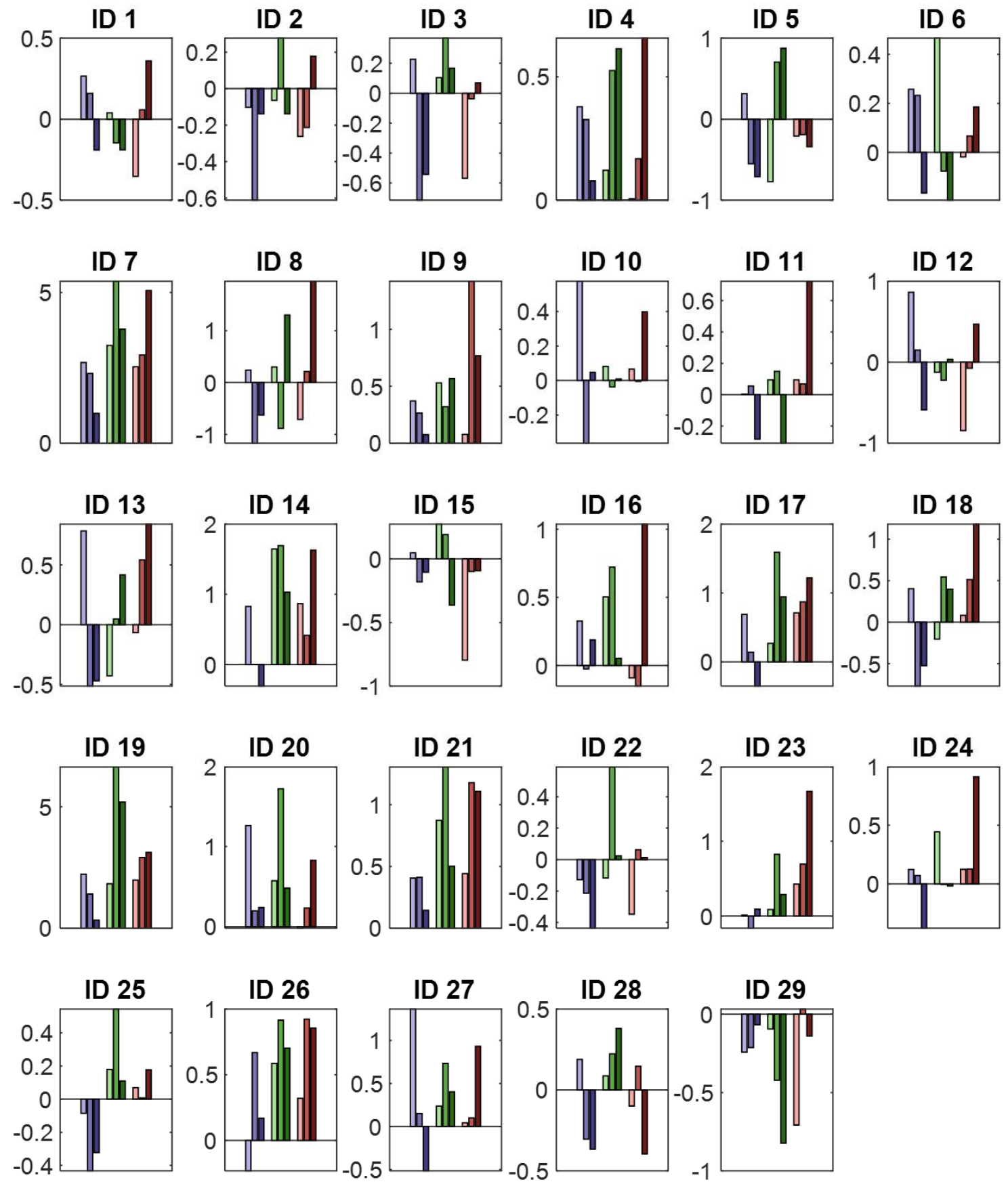

923

924

925

926

927

928

Figure 7-figure supplement 1. Power values for all conditions with a valid modality cue (expect heat receive heat) averaged over all significant time-frequency-electrode samples period for each subject (ID) of the negative absolute prediction error cluster. Blue colors represent low heat conditions, green colors represent medium heat conditions and red colors represent high heat conditions. Color intensities depict expectation level. 


\section{Stimulus Intensity}
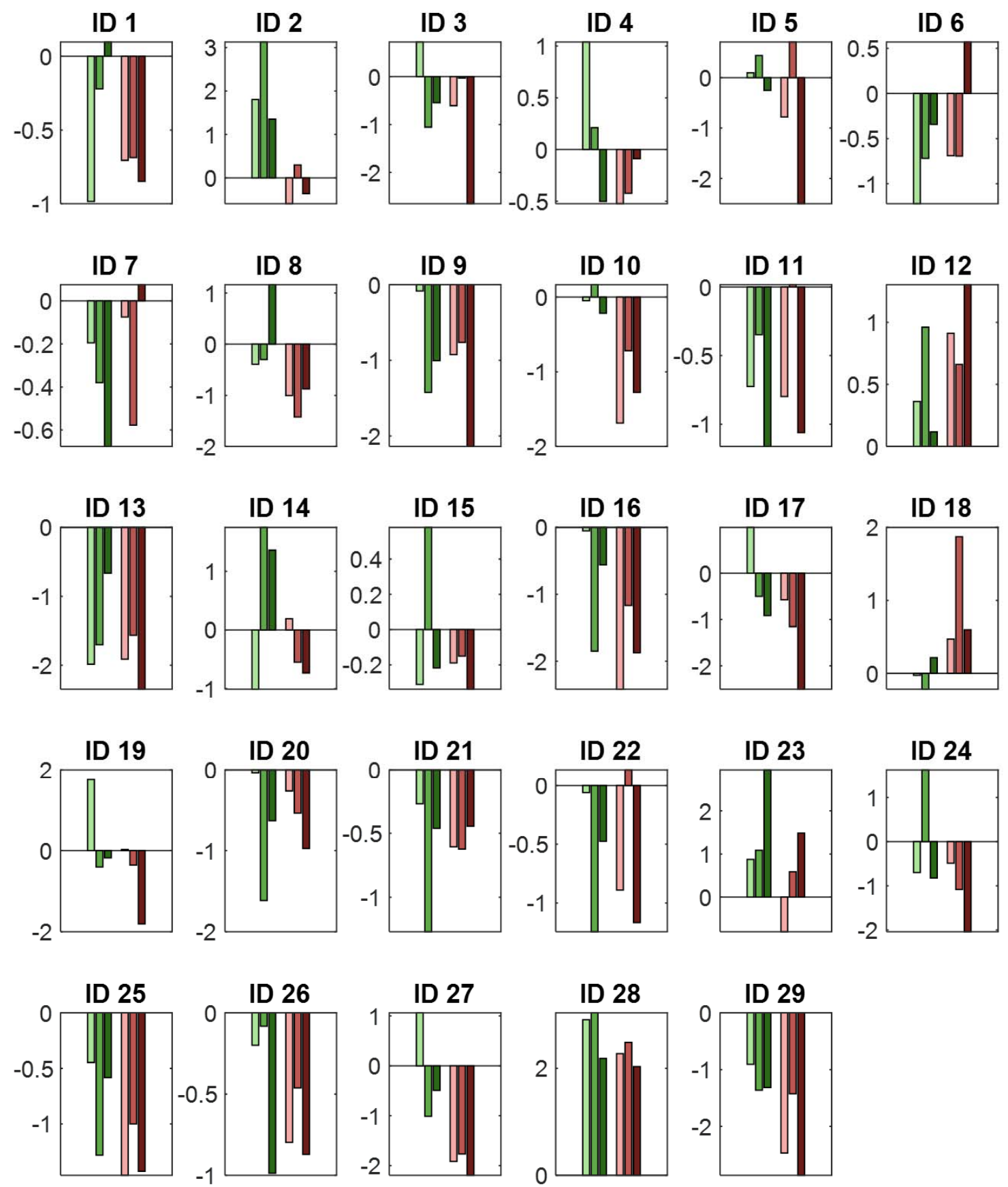

930

931 Figure 8-figure supplement 1. Power values for all medium and high intensity conditions with a valid modality cue (expect heat receive heat) averaged over all significant time-frequencyelectrode samples period for each subject (ID) of the negative stimulus intensity cluster of the analysis of the reduced model. Green colors represent medium heat conditions and red colors represent high heat conditions. Color intensities depict expectation level. 


\section{Neg. Absolute Prediction Error}
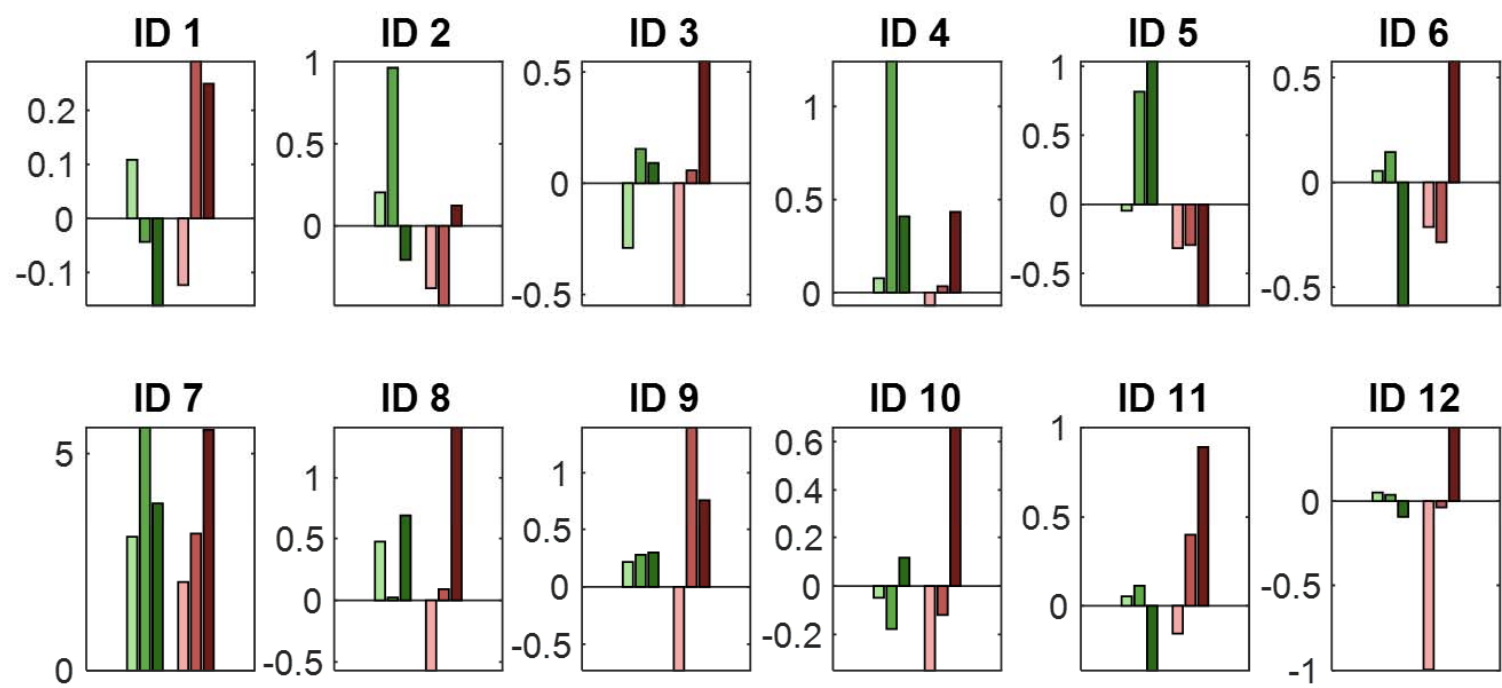

ID 12

ID 13

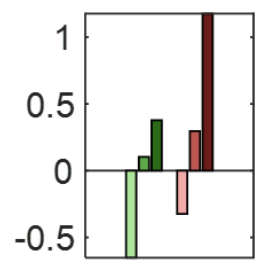

ID 14
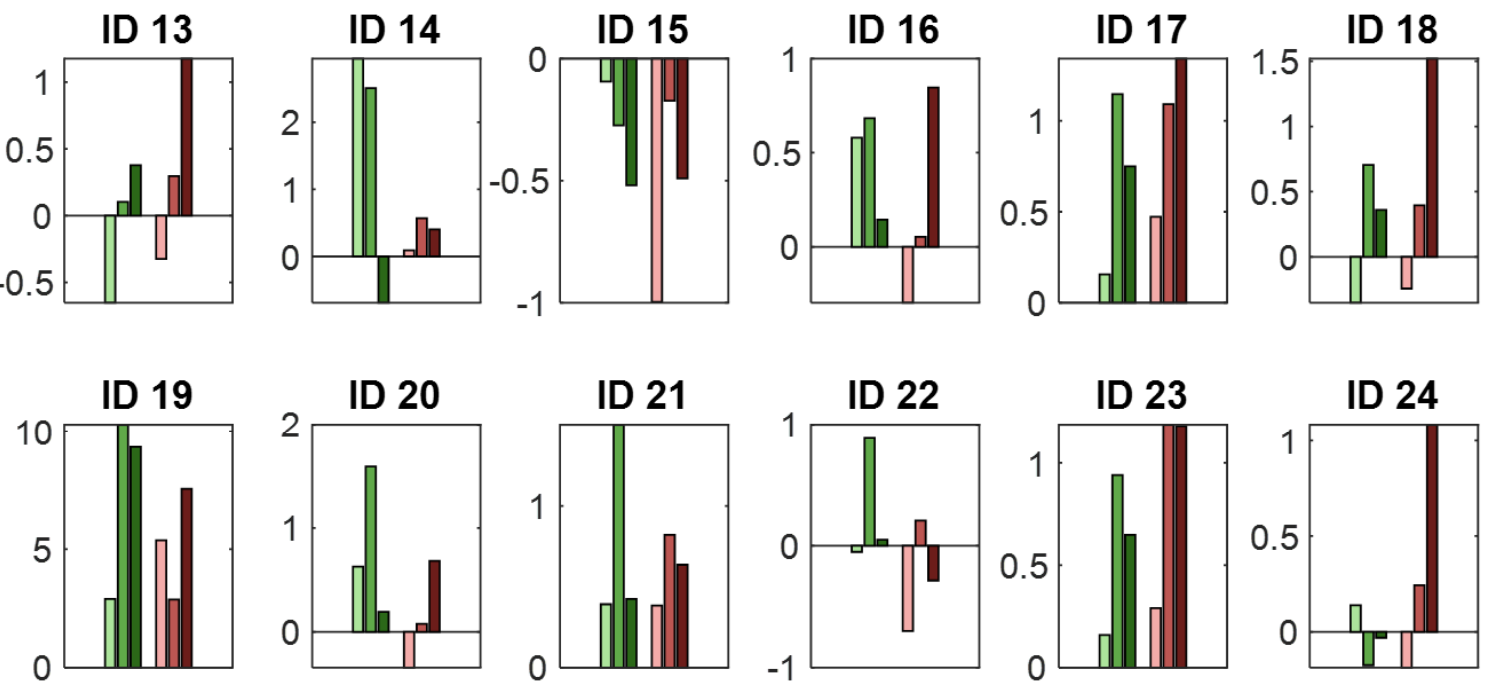

ID 24
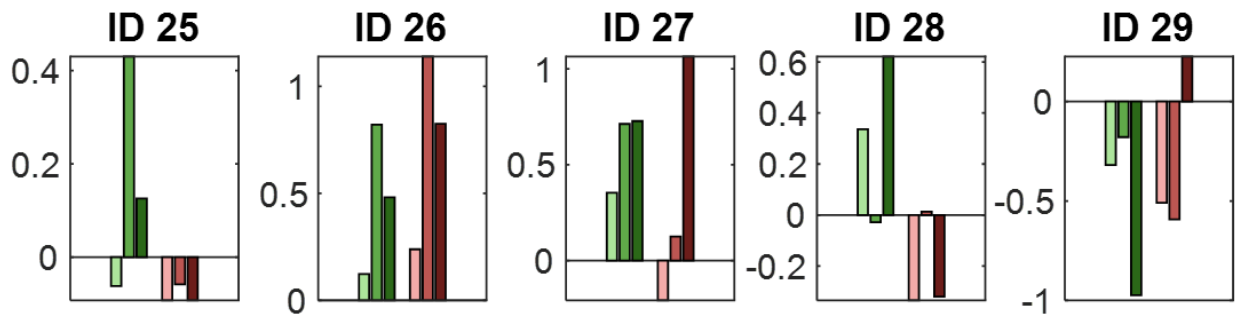

Figure 8-figure supplement 2. Power values for all medium and high intensity conditions with a valid modality cue (expect heat receive heat) averaged over all significant time-frequencyelectrode samples period for each subject (ID) of the negative absolute prediction error cluster of the analysis of the reduced model. Green colors represent medium heat conditions and red colors represent high heat conditions. Color intensities depict expectation level. 


\section{Expectation}
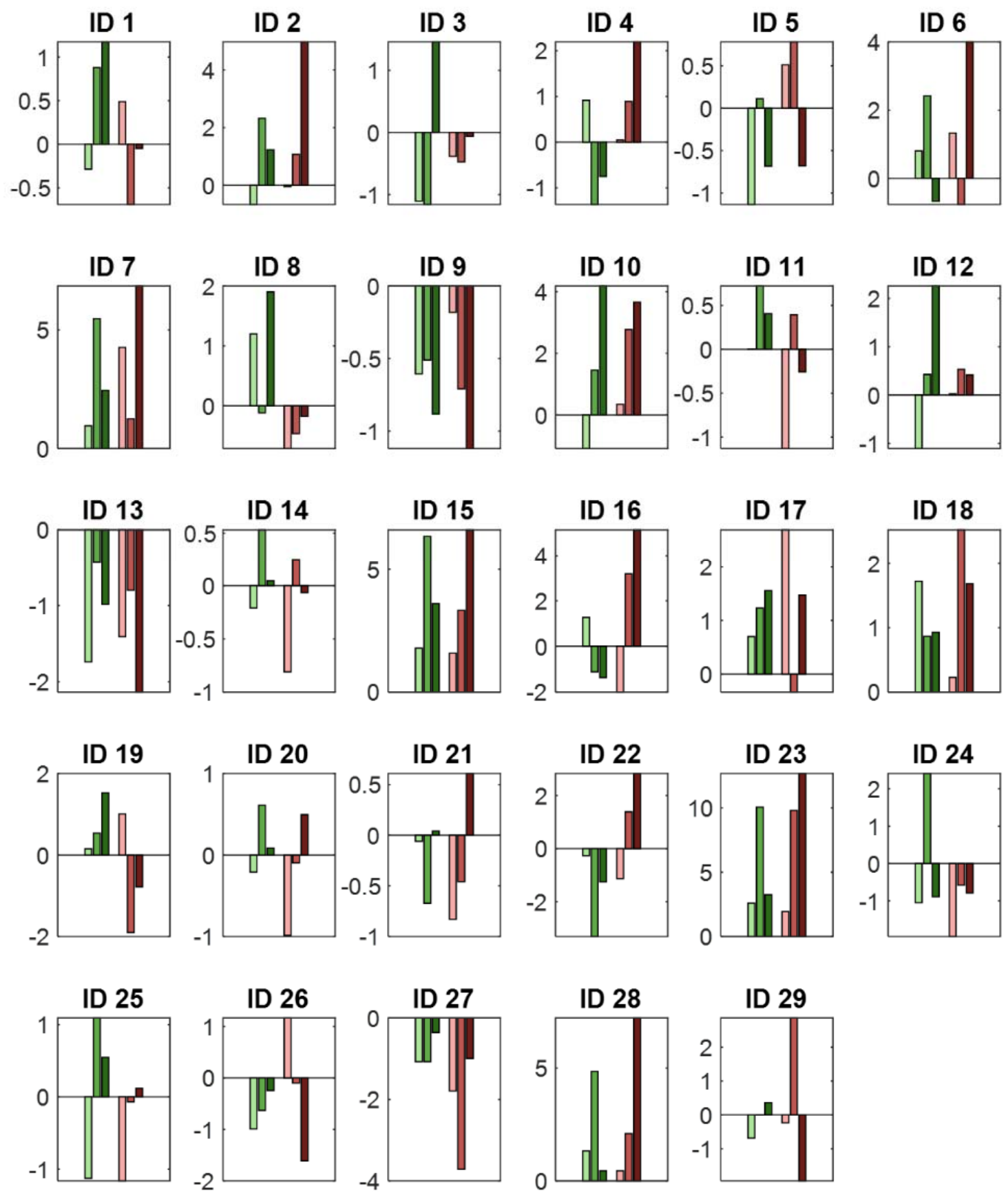

Figure 8-figure supplement 3. Power values for all medium and high intensity conditions with

944 a valid modality cue (expect heat receive heat) averaged over all (non-significant) time945 frequency-electrode samples of the respective cluster period for each subject (ID) of the non946 significant expectation cluster of the analysis of the reduced model. Green colors represent 947 medium heat conditions and red colors represent high heat conditions. Color intensities depict 948 expectation level. 